\title{
Resummation for Nonequilibrium Perturbation Theory and Application to Open Quantum Lattices
}

\author{
Andy C. Y. Li, ${ }^{1}$ F. Petruccione, ${ }^{2}$ and Jens Koch ${ }^{1,3}$ \\ ${ }^{1}$ Department of Physics and Astronomy, Northwestern University, Evanston, Illinois 60208, USA \\ ${ }^{2}$ Quantum Research Group, School of Chemistry and Physics, \\ University of KwaZulu-Natal, Durban 4001, South Africa \\ and National Institute for Theoretical Physics (NITheP), \\ KwaZulu-Natal, Durban 4001, South Africa \\ ${ }^{3}$ Kavli Institute for Theoretical Physics, University of California, \\ Santa Barbara, California 93106-4030, USA
}

(Received 19 November 2015; revised manuscript received 25 February 2016; published 16 June 2016)

Lattice models of fermions, bosons, and spins have long served to elucidate the essential physics of quantum phase transitions in a variety of systems. Generalizing such models to incorporate driving and dissipation has opened new vistas to investigate nonequilibrium phenomena and dissipative phase transitions in interacting many-body systems. We present a framework for the treatment of such open quantum lattices based on a resummation scheme for the Lindblad perturbation series. Employing a convenient diagrammatic representation, we utilize this method to obtain relevant observables for the open Jaynes-Cummings lattice, a model of special interest for open-system quantum simulation. We demonstrate that the resummation framework allows us to reliably predict observables for both finite and infinite Jaynes-Cummings lattices with different lattice geometries. The resummation of the Lindblad perturbation series can thus serve as a valuable tool in validating open quantum simulators, such as circuit-QED lattices, currently being investigated experimentally.

DOI: 10.1103/PhysRevX.6.021037

\section{INTRODUCTION}

Lattice models describe particles or spins residing on a set of sites, arranged in a regular fashion. Different types of interactions among these components are possible and can be included in the formulation of the model. For this reason, lattice models can cover a large arena of physical systems and phenomena. Prominent examples are the fermionic Hubbard model [1-3], the Bose-Hubbard model $[4,5]$, and the Heisenberg model [6,7].

Open quantum lattices extend the lattice-model concept. They include the effects of environment and external driving fields coupled to the lattice. Such open lattices generally describe nonequilibrium phenomena and are of great interest in many subfields of physics - ranging from condensed matter [8-10] and AMO physics [11-17] to applications in quantum information [18-23]. Recently, many studies of open quantum lattices have advanced our understanding of many-body systems under nonequilibrium conditions $[14,17,24]$. Examples of phenomena predicted to emerge in certain scenarios include nonequilibrium critical behavior [8,11,24-37], topological phases

Published by the American Physical Society under the terms of the Creative Commons Attribution 3.0 License. Further distribution of this work must maintain attribution to the author(s) and the published article's title, journal citation, and DOI.
Subject Areas: Condensed Matter Physics,

Quantum Physics, Quantum Information
$[15,16,38]$, and quantum chaos [17,39]. Open quantum lattices, especially with engineered coupling to baths, also play an increasingly vital role in the development of quantum information technology such as quantum computing hardware [18-20,23] and quantum networks [22].

The study of open quantum lattices tends to be challenging. Analytical and numerical techniques for open lattices are currently less developed than their counterparts for closed lattices. While for a large class of open quantum lattices the Lindblad master equation provides an appropriate theoretical framework [40,41], numerical methods for solving this master equation directly, such as exact diagonalization $[42,43]$, time evolution, or averaging of quantum trajectories [44], are computationally demanding and become quickly infeasible as lattice size increases. More sophisticated numerical techniques have been suggested and are further being developed, including matrix-product $[45,46]$, selfconsistent projection operator methods [47], and variational methods $[48,49]$. These methods can handle larger lattices to some degree but come with their own specific drawbacks.

As a result, the development of quantum simulators based on photons is particularly intriguing. Photonic systems represent an interesting open-system complement to the well-established paradigm of ultracold-atom quantum simulators. Since photons do not possess a chemical potential (however, see Ref. [50] for a proposal to engineer a chemical potential), realistic photonic lattices will 
typically include coherent driving and photon loss [51]. Such systems will thus be a particular useful tool to better understand, gain intuition, and ultimately devise tractable effective models for open quantum lattices of interest. Experiments with photonic quantum simulators will shed definitive light on both dynamical and steady-state phenomena by employing well-defined artificial lattice structures and systematically controlling parameters including drive strength, photon frequency, and strength of the mediated photon-photon interaction. Very promising experimental progress in this direction has already been made in the circuit QED architecture [51-53].

An experimental quantum simulator requires careful initial steps of validation [54] to ensure that the given physical system is correctly implementing the intended model. The validation procedure naturally demands that, for specific parameter regimes, theoretical understanding and reliable quantitative predictions are available and enable a comparison between theory and the experimental data obtained from the quantum simulator. For the purpose of validation, we utilize the well-controlled approximation scheme of nondegenerate Lindblad perturbation theory [28,55-58], which is not based on Keldysh Green's functions. We are particularly interested in the steady-state behavior of open lattices, which can be directly related to experimental observables such as microwave transmission in circuit-QED lattices [52,53]. Steady-state quantities are of paramount importance for the detection of dissipative phase transitions [11,24-26,28-30,32,34,36,59-63]. In the work presented here, we take a crucial step beyond finite-order perturbation theory by demonstrating a partial resummation of the perturbation series for the steady-state solution of the Lindblad master equation. We then employ this method to study an open Jaynes-Cummings (JC) lattice (Fig. 1) and establish that the resummation affords a significant improvement of the approximation accuracy. We illustrate the method's versatility in handling both finite-size and infinite lattices as well as different geometries and dimensionalities in a natural way. The method is hence well suited for validating data from the first circuit-QED quantum simulators currently being investigated [64].

Our discussion is structured as follows. We set the stage with a general review of (Markovian) open quantum lattices in Sec. II, examining their theoretical description in terms of the Lindblad master equation. Section III forms the centerpiece of our theoretical framework: Here, we introduce the resummation scheme by which we can include certain perturbative corrections up to infinite order, and formally show its validity. We then apply this technique to the open JC lattice model in Sec. IV. After a few preparatory steps (Sec. IV A) and including use of third-quantization methods [65-72] to obtain an exact solution in the absence of perturbation (Sec. IV B), we perform the resummation and obtain results for steady-state observables (Sec. IV C). We finally compare our results to both finite-order perturbation theory and exact solutions (where available) and discuss the role of finite-size effects and lattice structures (Sec. IV D). We conclude with a summary in Sec. V.

\section{BACKGROUND: LINDBLAD FORMALISM FOR DRIVEN, DISSIPATIVE LATTICES}

Open quantum lattice models are widely used to study many-body physics under nonequilibrium conditions. There exists a large variety of such lattice models, including open photon lattices [12,13,73], Jaynes-Cummings lattices [26,29,30,62,74,75], Dicke-type models [24,25,76-78], and lattices with different types of interactions $[33,79,80]$. Open lattices are not limited to bosons but may also involve fermions and spins and may include both on-site and off-site interactions. We denote the Hamiltonians governing the unitary evolution from on-site and off-site terms by $h_{r}$ and $\mathrm{V}_{\mathbf{r r}}$, respectively. The resulting generic system Hamiltonian is then given by

$$
\mathrm{H}=\sum_{\mathbf{r}} \mathrm{h}_{\mathbf{r}}+\sum_{\mathbf{r} \neq \mathbf{r}^{\prime}} \mathrm{V}_{\mathbf{r r}^{\prime}}
$$
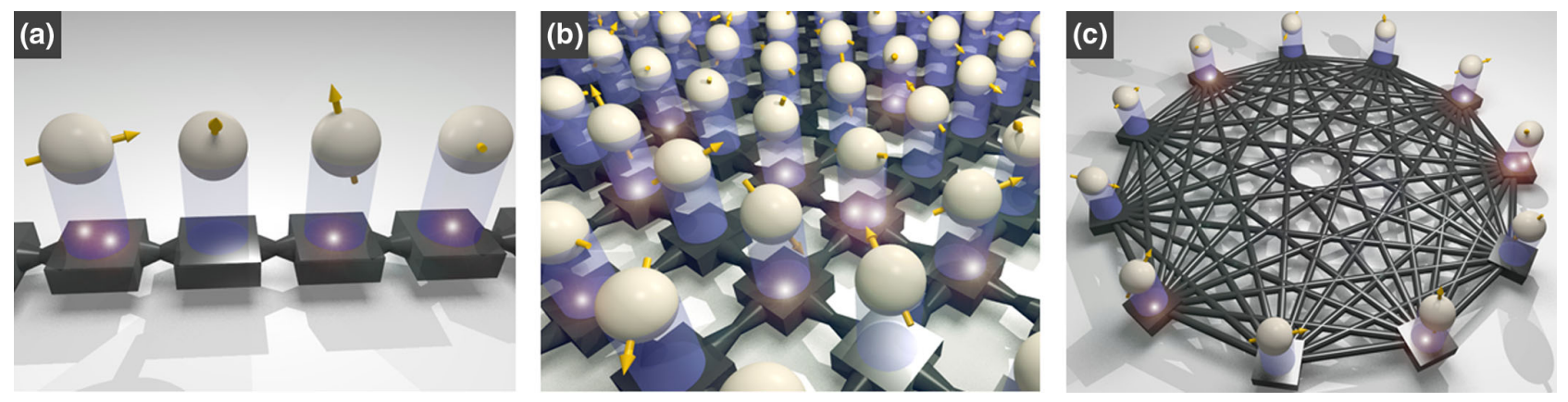

FIG. 1. Open quantum lattices of different dimensionalities and geometries. The examples show Jaynes-Cummings lattices in which photons can hop between neighboring resonators (dark boxes) and experience an interaction mediated by the coupling to two-level systems (represented as spin systems). Lattice types of interest include (a) one-dimensional Jaynes-Cummings chains, (b) two-dimensional arrays such as the depicted square lattice, and (c) more artificial arrangements of theoretical interest, such as the global-coupling scenario where each site is connected to all other sites. 
In many cases, off-site interactions can be limited to nearestneighbor pairs $\left\langle\mathbf{r}, \mathbf{r}^{\prime}\right\rangle$.

For an open quantum lattice, we further account for the coupling to environmental degrees of freedom. Considering the effective dynamics of the lattice only, one finds that the environment generally induces nonunitary evolution, which cannot be captured by an effective lattice Hamiltonian. The environment induces effects such as dissipation and decoherence, so the time evolution of the reduced density matrix of the lattice $\rho$ generally deviates from the unitary von Neumann equation $\dot{\rho}=-i[\mathrm{H}, \rho]$.

In many relevant cases of weak coupling to the environment, the lattice system will undergo Markovian dynamics: The state of the system at the current time $t$ fully determines the state at the slightly advanced time $t+d t$ in the future. (Another way to express this is to say that there are no "memory effects.") Under a fairly general set of conditions [40], the time evolution of $\rho$ is then described by the Lindblad master equation [40,41],

$$
\dot{\rho}=-i[\mathrm{H}, \rho]+\sum_{j} \gamma_{j} \mathbb{D}\left[\mathbf{f}_{j}\right] \rho .
$$

The influence of the environment is thus encoded in the damping terms $\sum_{j} \gamma_{j} \mathbb{D}\left[\mathbf{f}_{j}\right]$, where $\mathbb{D}\left[\mathbf{f}_{j}\right] \rho \equiv \mathbf{f}_{j} \rho \mathbf{f}_{j}^{\dagger}-\mathbf{f}_{j}^{\dagger} \mathrm{f}_{j} \rho / 2-$ $\rho \mathrm{f}_{j}^{\dagger} \mathrm{f}_{j} / 2$ is called the dissipator. Typically, each particular jump operator $f_{j}$ points to a particular nonunitary process. For example, photon decay commonly results from the photon annihilation operator a acting as the jump operator. (This statement is oversimplifying matters somewhat. In general, care must be taken to derive the appropriate jump operator for a particular system and environment coupling $[40,81]$.) The prefactor $\gamma_{j}$ characterizes the rate of the damping process.

As a concrete example of an open quantum lattice and paradigm for interacting photon lattices, we consider the driven, damped Jaynes-Cummings lattice (Fig. 2). In this system, each site consists of a harmonic oscillator, such as the mode of an electromagnetic resonator, coupled coherently to a two-level system, referred to as "qubit" in the following. Each site can be driven by a coherent tone. For simplicity, we consider the situation of a global drive frequency $\omega_{d}$, identical on each site.

The single-site Hamiltonian for this lattice is the JaynesCummings Hamiltonian plus drive term,

$$
\begin{aligned}
\mathrm{h}_{\mathbf{r}}= & \delta \omega_{\mathbf{r}} \mathrm{a}_{\mathbf{r}}^{\dagger} \mathrm{a}_{\mathbf{r}}+\delta \Omega_{\mathbf{r}} \sigma_{\mathbf{r}}^{+} \sigma_{\mathbf{r}}^{-}+g_{\mathbf{r}}\left(\mathrm{a}_{\mathbf{r}} \sigma_{\mathbf{r}}^{+}+\mathrm{a}_{\mathbf{r}}^{\dagger} \sigma_{\mathbf{r}}^{-}\right) \\
& +\epsilon_{\mathbf{r}}\left(\mathrm{a}_{\mathbf{r}}+\mathrm{a}_{\mathbf{r}}^{\dagger}\right) .
\end{aligned}
$$

In the usual way, we have already eliminated the original time dependence of the drive by switching to a frame rotating with the drive frequency. Consequently, the photon and qubit terms involve frequency detunings relative to the drive. Specifically, we have $\delta \omega_{\mathbf{r}} \equiv \omega_{\mathbf{r}}-\omega_{d}$ for the photon

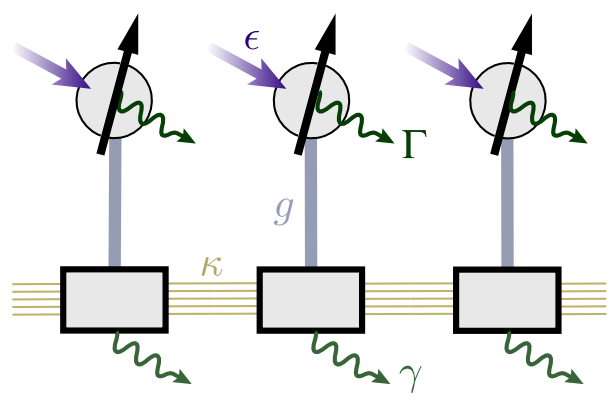

FIG. 2. Constituents of the Jaynes-Cummings lattice: Two-level systems are driven coherently with strength $\epsilon$ and exchange excitations with local harmonic oscillators at a rate set by $g$. Together, a pair of two-level system and oscillator form one site of the Jaynes-Cummings system. Nearest-neighbor sites are coupled by propagation of oscillator excitations with rate $\sim \kappa$. Dissipation is included in the form of two-level relaxation (rate $\Gamma$ ) and oscillator relaxation (rate $\gamma$ ). In the circuit-QED realization, two-level systems are implemented by superconducting qubits, and oscillators by photon modes of on-chip microwave resonators with typical frequencies in the range of a few $\mathrm{GHz}$.

mode with frequency $\omega_{\mathbf{r}}$, and $\delta \Omega_{\mathrm{r}} \equiv \Omega_{\mathrm{r}}-\omega_{d}$ for the qubit with frequency $\Omega_{\mathbf{r}}$ on site $\mathbf{r}$. Photon and qubit excitations are created (annihilated) by the standard ladder operators $\mathrm{a}_{\mathbf{r}}^{\dagger}$ and $\sigma_{\mathbf{r}}^{+}\left(\mathrm{a}_{\mathbf{r}}\right.$ and $\left.\sigma_{\mathbf{r}}^{-}\right)$, respectively. We denote the strengths of the Jaynes-Cummings coupling and of the coherent drive tone by $g_{\mathbf{r}}$ and $\epsilon_{\mathbf{r}}$. Off-site terms of this lattice arise from the hopping of photons between sites $\mathbf{r}$ and $\mathbf{r}^{\prime}$ with rate $\kappa_{\mathbf{r r}}$ :

$$
\mathrm{V}_{\mathbf{r r}^{\prime}}=\kappa_{\mathbf{r r}^{\prime}}\left(\mathrm{a}_{\mathbf{r}}^{\dagger} \mathrm{a}_{\mathbf{r}^{\prime}}+\mathrm{a}_{\mathbf{r}} \mathrm{a}_{\mathbf{r}^{\prime}}^{\dagger}\right) .
$$

The full Hamiltonian consists of the appropriate sum of on-site and off-site contributions (as given above).

Finally, we consider the damping induced by photon decay $\left(\right.$ rate $\gamma_{\mathbf{r}}$ ) and qubit relaxation $\left(\right.$ rate $\Gamma_{\mathbf{r}}$ ). If both processes occur because of coupling to separate zerotemperature baths, then the appropriate jump operators can be shown to be the photon annihilation operator $a_{r}$ and the pseudospin lowering operator $\sigma_{\mathbf{r}}^{-}$. Overall, we thus obtain the Lindblad master equation

$$
\dot{\rho}=-i[\mathrm{H}, \rho]+\sum_{\mathbf{r}} \gamma_{\mathbf{r}} \mathbb{D}\left[\mathbf{a}_{\mathbf{r}}\right] \rho+\sum_{\mathbf{r}} \Gamma_{\mathbf{r}} \mathbb{D}\left[\sigma_{\mathbf{r}}^{-}\right] \rho .
$$

We frequently find it convenient to write the Lindblad master equation in the short form $\dot{\rho}=\mathbb{L} \rho$. Here, $\mathbb{L}$ is the socalled Liouville superoperator. The term "superoperators" designates an object mapping an ordinary Hilbert-space operator such as $\rho$ to another Hilbert-space operator. (In our notation, we distinguish superoperators, operators, and real/complex numbers by using double-stroke, sans-serif, and regular lettering, respectively.) Using this shorthand of the master equation, we can easily characterize the steady state $\rho_{\mathrm{s}}$ of the lattice: It is the stationary solution of this Lindblad master equation, i.e., 


$$
\dot{\rho}_{\mathrm{s}}=\mathbb{L} \rho_{\mathrm{s}}=0,
$$

with normalization $\operatorname{tr} \rho_{\mathrm{s}}=1$.

Mathematically, the equation $\mathbb{L} \rho_{\mathrm{s}}=0$ is a system of linear equations for the components of $\rho_{\mathrm{s}}$. It can also be interpreted as a special instance of the eigenvalue problem for the Liouville superoperator $\mathbb{L}$,

$$
\mathbb{L} \mathrm{u}_{\mu}=\lambda_{\mu} \mathrm{u}_{\mu},
$$

namely, as the instance of eigenvalue $\lambda_{\mu}=0$. (We always take $\mu=0$ to denote this case in the following.) The steady state $\rho_{\mathrm{s}}$ is thus the eigenstate $\mathrm{u}_{0}=\rho_{\mathrm{s}}$ of $\mathbb{L}$ associated with the eigenvalue $\lambda_{0}=0$. While it is tempting to think of Eq. (7) as a superoperator analogue of the stationary Schrödinger equation, it is important to note that the Liouville superoperator $\mathbb{L}$ is, in general, not Hermitian; i.e., $\mathbb{L}^{\dagger}$ is not equal to $\mathbb{L}$. Hence, its eigenvalues may be complex valued, and we have to distinguish right eigenstates $\mathrm{u}_{\mu}$ from left eigenstates $\breve{\mathrm{u}}_{\mu}$, given by

$$
\breve{\mathrm{u}}_{\mu}^{\dagger} \mathbb{L}=\breve{\mathrm{u}}_{\mu}^{\dagger} \lambda_{\mu} \text {. }
$$

As long as we keep this in mind, however, it is useful to mimic bra-ket notation and allow ourselves the freedom to write operators and their adjoints also in the alternative form $\left.\mathrm{u}_{\mu} \leftrightarrow \mid \mathrm{u}_{\mu}\right)$ and $\breve{\mathrm{u}}_{\mu}^{\dagger} \leftrightarrow\left(\breve{\mathrm{u}}_{\mu} \mid\right.$. We can then denote the Hilbert-Schmidt inner product between operators as $(\mathbf{x} \mid \mathbf{y}) \equiv \operatorname{tr}\left[\mathbf{X}^{\dagger} \mathbf{y}\right]$. Linear algebra dictates that the right and left eigenstates of $\mathbb{L}$ are orthogonal and, by appropriate normalization, can be chosen to be orthonormal,

$$
\left(\breve{\mathrm{u}}_{\nu} \mid \mathrm{u}_{\mu}\right)=\delta_{\mu \nu} .
$$

Assuming that the eigenstates of $\mathbb{L}$ form a complete set [82], we can represent an arbitrary operator $\mathbf{X}$ as

$$
\left.\left.\mid \mathbf{X})=\sum_{\mu} \mid \mathbf{u}_{\mu}\right)\left(\breve{\mathbf{u}}_{\mu} \mid \mathbf{X}\right)=\sum_{\mu}(\mathbf{X})_{\mu} \mid \mathbf{u}_{\mu}\right)
$$

and an arbitrary superoperator $\mathbb{A}$ as

$$
\left.\mathbb{A}=\sum_{\mu, \nu} \mid \mathrm{u}_{\mu}\right)\left(\breve{\mathrm{u}}_{\mu}|\mathbb{A}| \mathrm{u}_{\nu}\right)\left(\breve{\mathrm{u}}_{\nu}\left|=\sum_{\mu, \nu}(\mathbb{A})_{\mu \nu}\right| \mathrm{u}_{\mu}\right)\left(\breve{\mathrm{u}}_{\nu} \mid .\right.
$$

Except for the matter of left vs right eigenvectors, these expressions are familiar from the usual decomposition of states and operators in Hilbert space, and we will make use of them in Sec. III.

\section{LINDBLAD PERTURBATION THEORY AND RESUMMATION}

In this section, we present the formalism of Lindblad perturbation theory and its resummation. This section remains general and is applicable to Markovian open quantum systems of various types. The concrete application of the formalism to an open Jaynes-Cummings lattice follows in Sec. IV. Together, these two sections constitute the central result of our paper.

Consider the general case of an open quantum system with Hamiltonian $\mathrm{H}$ and Liouville superoperator $\mathbb{L}$. We assume that $\mathbb{L}$ is amenable to a perturbative treatment and can be decomposed into a sum $\mathbb{L}=\mathbb{L}_{0}+\mathbb{L}_{1}$, consisting of the unperturbed Liouville superoperator $\mathbb{L}_{0}$ and the perturbation $\mathbb{L}_{1}$. For $\mathbb{L}_{0}$ to qualify as such, it is expected that we can obtain its spectrum exactly. We denote the resulting unperturbed eigenvalues by $\lambda_{\mu}^{0}$ and the corresponding unperturbed right and left eigenstates by $\left.\mid \mathbf{u}_{\mu}^{0}\right)$ and $\left(\breve{u}_{\mu}^{0} \mid\right.$, respectively.

The spectra of $\mathbb{L}$ and $\mathbb{L}_{0}$ differ, but corrections may be calculated by a perturbative series expansion if $\mathbb{L}_{1}$ is "sufficiently small." The corrections to eigenvalues and eigenstates can then be determined recursively, order by order [58]. Our interest here primarily regards the steady state $\rho_{\mathrm{s}}$, and we apply Lindblad perturbation theory assuming the nondegenerate case in which the steady state is unique. Two remarks may be useful for clarification. First, we emphasize that nondegeneracy refers to the spectrum of $\mathbb{L}_{0}$, not to the Hamiltonian $\mathrm{H}$; we make no assumptions about the spectrum of $\mathrm{H}$. Second, we note that nonuniqueness of the steady state and resulting nonanalyticities are crucial at the phase boundary of a dissipative phase transition. Perturbative series expansions will generally not hold directly at such a boundary but may still be applicable in its vicinity.

Turning now to the concrete series expansion $\left.\mid \rho_{\mathrm{s}}\right)=$ $\left.\sum_{j} \mid \rho_{j}\right)$ of the steady state, we note that the $j$ th order contribution $\rho_{j}$ is obtained from the recursion relation

$$
\left.\left.\mid \rho_{j}\right)=-\mathbb{L}_{0}^{-1} \mathbb{L}_{1} \mid \rho_{j-1}\right) .
$$

Here, inversion of $\mathbb{L}_{0}$ will always be understood as restricted to the space orthogonal [83] to the unperturbed steady state, i.e., $\left.\mathbb{L}_{0}^{-1}=\sum_{\mu>0}\left(\lambda_{\mu}^{0}\right)^{-1} \mid \mathbf{u}_{\mu}\right)\left(\breve{\mathbf{u}}_{\mu} \mid\right.$. With this, we obtain the formal expression

$$
\left.\left.\left.\mid \rho_{j}\right)=\left(-\mathbb{L}_{0}^{-1} \mathbb{L}_{1}\right)^{j} \mid \rho_{0}\right)=\mathbb{U}^{j} \mid \rho_{0}\right),
$$

where $\rho_{0}$ is the unperturbed steady state of $\mathbb{L}_{0}$, and we

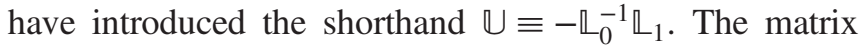
elements of the $\mathbb{U}$ superoperator are

$$
(\mathbb{U})_{\mu \nu}=\left(\breve{\mathbf{u}}_{\mu}^{0}|\mathbb{U}| \mathbf{u}_{\nu}^{0}\right)=-\left(\breve{\mathbf{u}}_{\mu}^{0}\left|\mathbb{L}_{1}\right| \mathbf{u}_{\nu}^{0}\right) / \lambda_{\mu}^{0}
$$

for $\mu>0$ and $(\mathbb{U})_{0 \nu}=0$. Using this shorthand, we write the $j$ th order contribution to the steady state in the form of the chain expression 


$$
\left.\left.\mid \rho_{j}\right)=\sum_{\mu_{1}, \mu_{2}, \ldots, \mu_{j}} \mid u_{\mu_{j}}^{0}\right) \cdot(\mathbb{U})_{\mu_{j} \mu_{j-1}} \cdots(\mathbb{U})_{\mu_{2} \mu_{1}}(\mathbb{U})_{\mu_{1} 0}
$$

and represent it diagrammatically as shown in Fig. 3(a). As a diagrammatic rule, we choose dots to represent unperturbed eigenstates, $\left.\mid \mathbf{u}_{\mu_{j}}^{0}\right)$, and interconnecting lines to represent factors of $\mathbb{U}$. Reading from the left to right, the leftmost state is the unperturbed steady state $\left.\left.\mid \rho_{0}\right)=\mid \mathbf{u}_{0}^{0}\right)$, and the rightmost one the final state $\left(\rho_{\mu_{j}}\right)$ which appears explicitly in the expression (15). All intermediate states and the final state, $\mu_{1}$ through $\mu_{j}$, are subject to summation but cannot coincide with the initial unperturbed steady state because of $(\mathbb{U})_{0 \nu}=0$.

To facilitate our resummation scheme for the steady-state series

$$
\left.\left.\mid \rho_{\mathrm{s}}\right)=\sum_{j=0}^{\infty} \mathbb{U}^{j} \mid \rho_{0}\right),
$$

we decompose the $\mathbb{U}$ superoperator into two parts

$$
\mathbb{U}^{1}=\mathbb{S}_{1}+\mathbb{T}_{1}
$$

To make the definition parallel to expressions to come, we have explicitly recorded the exponent $j=1$ on the lefthand side. We now specify the terms on the right-hand side

(a)

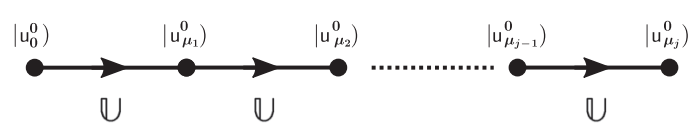

(b)

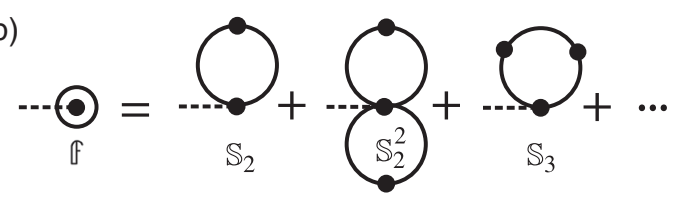

(c)

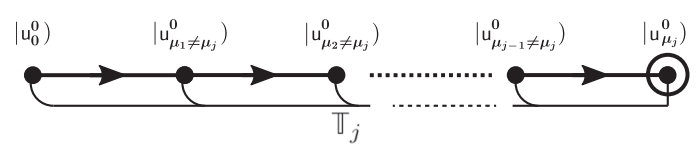

FIG. 3. Diagrams for perturbative corrections. (a) The order- $j$ correction to the steady state [Eq. (15)] is depicted as a chain of $j$ lines, each representing one factor $\mathbb{U}$. The chain connects $j+1$ dots symbolizing $\mathbb{L}_{0}$ eigenstates. The leftmost state is the unperturbed steady state $\left.\left|\rho_{j}\right|=\mid u_{0}^{0}\right)$, the rightmost one the unperturbed eigenstate $\left.\mid u_{\mu_{j}}^{0}\right)$, giving rise to one specific correction term. The full correction is obtained by summation over final and intermediate states $\mu_{1}, \ldots, \mu_{j}$. (b) The resummation superoperator $\mathbb{f}$ is the sum of all (reducible and irreducible) $\mathbb{S}$ superoperators. Each $\mathbb{S}$ diagram starts and ends with the same unperturbed eigenstate. (c) Resummation combines evaluation of $\mathbb{S}$ and $\mathbb{T}$ superoperators. Terms of rank $j$ are comprised of the fully off-diagonal chain specified by $\mathbb{T}_{j}$ and final application of the resummation superoperator. in such a way that resummation takes a particular simple form. We define the first-order $\mathbb{S}$ superoperator as the diagonal part of $\mathbb{U}^{1}$, i.e., $\left(\mathbb{S}_{1}\right)_{\mu \nu}=\delta_{\mu \nu}\left(\mathbb{U}^{1}\right)_{\mu \nu}$. Accordingly, $\mathbb{S}_{1}$ and $\mathbb{L}_{0}$ share the same set of right eigenstates, i.e., $\left.\left.\mathbb{S}_{1} \mid \mathbf{u}_{\mu}^{0}\right)=\Sigma_{1 ; \mu} \mid \mathbf{u}_{\mu}^{0}\right)$, and the eigenvalue is $\Sigma_{1 ; \mu}=\left(\mathbb{U}^{1}\right)_{\mu \mu}$. We see that this simplicity of $\mathbb{S}_{1}$ will be important for the resummation of the series, Eq. (16). The term $\mathbb{T}_{1}$ in Eq. (17) is the off-diagonal remainder of the $\mathbb{U}$ superoperator.

A simplification of the previous expressions occurs when making the natural assumption that the perturbation $\mathbb{L}_{1}$ is itself off diagonal with respect to the unperturbed eigenstates of $\mathbb{L}_{0}$. (Whenever $\mathbb{L}_{1}$ does not satisfy this assumption, a simple redefinition of $\mathbb{L}_{0}$ and $\mathbb{L}_{1}$ can be used to turn $\mathbb{L}_{1}$ off diagonal.) Now, if $\mathbb{L}_{1}$ is off diagonal, so is $\mathbb{U}=-\mathbb{L}_{0}^{-1} \mathbb{L}_{1}$, and we immediately obtain

$$
\mathbb{S}_{1}=0 \quad \text { and } \quad \mathbb{T}_{1}=\mathbb{U} .
$$

This may initially make the decomposition of $\mathbb{U}$ seem pointless, but we will see momentarily that this simplification does not carry over to higher orders $j>1$, thus justifying our approach.

We next consider the second-order term $\left.\mathbb{U}^{2} \mid \rho_{0}\right)$, which warrants a decomposition of $\mathbb{U}^{2}=\mathbb{V}_{1} \mathbb{U}$ into

$$
\mathbb{T}_{1} \mathbb{U}=\mathbb{S}_{2}+\mathbb{T}_{2} .
$$

Analogous to our strategy above, we define the secondorder $\mathbb{S}$ superoperator, as the diagonal part of the left-hand side,

$$
\left(\mathbb{S}_{2}\right)_{\mu \nu}=\delta_{\mu \nu}\left(\mathbb{T}_{1} \mathbb{U}\right)_{\mu \nu}=\delta_{\mu \nu} \sum_{\tau}(\mathbb{U})_{\nu \tau}(\mathbb{U})_{\tau \nu} .
$$

Note that the off-diagonal character of $\mathbb{U}$ automatically leads to exclusion of the term with $\tau=\nu$. As before, $\mathbb{T}_{2}$ represents the remaining off-diagonal part in Eq. (19). We represent $\mathbb{S}_{2}$ by the loop diagram shown in Fig. 3(b). Since $\mathbb{S}_{2}$ is diagonal, its initial and final states $\left.\mid \mathbf{u}_{\mu}^{0}\right)$ must be identical. However, the intermediate state $\left.\mid \mathbf{u}_{\tau}^{0}\right)$ involved in the expression (20) must differ from $\mid u_{\mu}^{0}$ ).

For resummation of terms to infinite order, we need to formulate our decomposition strategy for arbitrary order $j$. It is natural to extend the definitions for diagonal and offdiagonal superoperators, Eqs. (19) and (20), by setting

$$
\mathbb{T}_{j-1} \mathbb{U}=\mathbb{S}_{j}+\mathbb{T}_{j}, \quad\left(\mathbb{S}_{j}\right)_{\mu \nu}=\delta_{\mu \nu}\left(\mathbb{T}_{j-1} \mathbb{U}\right)_{\mu \nu} .
$$

The recurrence relation is solved by

$$
\mathbb{T}_{j}=\llbracket \llbracket \cdots \llbracket \llbracket \mathbb{U} \rrbracket \mathbb{U} \rrbracket \cdots \mathbb{U} \rrbracket \mathbb{U} \rrbracket \quad(j \text { times }),
$$

where $\llbracket \mathbb{A} \rrbracket$ denotes the off-diagonal part of $\mathbb{A}$ with respect to the unperturbed basis $\left.\left\{\mid \mathbf{u}_{\mu}^{0}\right)\right\}$. We must note, however, that the definition (21) does not yet determine a unique 
separation scheme beyond second order. Consider, for instance, the case of the third-order term involving $\mathbb{U}^{3}$. While we know the decomposition of $\mathbb{U}^{2}=\mathbb{T}_{1} \mathbb{U}$ from Eq. (19), we still have the freedom to perform the substitution for either $\mathbb{U}^{3}=\mathbb{U}\left(\mathbb{U}^{2}\right)$ or $\mathbb{U}^{3}=\left(\mathbb{U}^{2}\right) \mathbb{U}$. Both forms are mathematically equivalent, but only the systematic usage of one replacement rule produces expressions for which resummation becomes simple. We consistently employ the form

$$
\mathbb{U}^{j}=\mathbb{U}^{j-1} \mathbb{U}
$$

where $\mathbb{U}^{j-1}$ signals that $\mathbb{U}^{j-1}$ is to be replaced by an expression composed of $\mathbb{S}, \mathbb{T}$, and $\mathbb{U}$ superoperators. Multiple replacements, in some cases making use of the identity $\mathbb{U}=\mathbb{T}_{1}$, may be necessary to reach the final decomposed form only involving $\mathbb{S}$ and $\mathbb{T}$ superoperators.

For illustration, we consider the decompositions of $\mathbb{U}^{3}$, $\mathbb{U}^{4}$, and $\mathbb{U}^{5}$. For the third-order case, we first make use of Eq. (23) and then Eq. (21) to obtain

$$
\mathbb{U}^{3}=\mathbb{U}^{2} \mathbb{U}=\mathbb{\mathbb { T }}_{1} \mathbb{U} \mathbb{U}=\mathbb{\mathbb { T }}_{2} \mathbb{U}+\mathbb{S}_{2} \mathbb{U} .
$$

The last term on the right-hand side cannot be simplified further (except for substituting $\mathbb{U}=\mathbb{T}_{1}$ ); the first term is further decomposed by using Eq. (21), leading to the final expression

$$
\mathbb{U}^{3}=\mathbb{\mathbb { T }}_{2} \mathbb{U}+\mathbb{S}_{2} \mathbb{\mathbb { T }}_{1}=\mathbb{S}_{3}+\mathbb{\mathbb { T }}_{3}+\mathbb{S}_{2} \mathbb{\mathbb { T }}_{1} .
$$

For the fourth order, we merely sketch the decomposition,

$$
\begin{aligned}
\mathbb{U}^{4} & =\underbrace{\mathbb{U}^{3} \mathbb{U}}=\mathbb{S}_{2} \mathbb{\mathbb { T }}_{1} \mathbb{U}+\mathbb{S}_{3} \mathbb{U}+\mathbb{S}_{4}+\mathbb{T}_{4} \\
& =\mathbb{S}_{4}+\mathbb{S}_{2}^{2}+\mathbb{S}_{3} \mathbb{\mathbb { T }}_{1}+\mathbb{S}_{2} \mathbb{\mathbb { T }}_{2}+\mathbb{\mathbb { T }}_{4} .
\end{aligned}
$$

We give the fifth-order result without showing substeps,

$$
\begin{aligned}
\mathbb{U}^{5}= & \mathbb{S}_{5}+\mathbb{S}_{2} \mathbb{S}_{3}+\mathbb{S}_{3} \mathbb{S}_{2} \\
& +\left(\mathbb{S}_{4}+\mathbb{S}_{2}^{2}\right) \mathbb{\mathbb { T }}_{1}+\mathbb{S}_{3} \mathbb{\mathbb { T }}_{2}+\mathbb{S}_{2} \mathbb{\mathbb { T }}_{3}+\mathbb{T}_{5} .
\end{aligned}
$$

Inspection of Eqs. (25)-(27) indicates a systematic structure underlying the expressions, namely,

$$
\mathbb{U}^{j}=\sum_{k=0}^{j} \mathbb{S}_{j-k} \mathbb{\mathbb { T }}_{k} .
$$

Each term in this sum has one factor of $\mathbb{T}_{k}$ of order $0 \leq$ $k \leq j$ and a prefactor $\mathbb{s}_{j-k}$ consisting of all possible combinations of $\mathbb{S}$ superoperators of total order $j-k$. A formal proof of this is given in Appendix A. [Recall from Eq. (18) that $\mathbb{S}_{1}=0$, which reduces the number of terms significantly.] Using the decomposition (28) and regrouping terms according to each occurrence of $\mathbb{T}_{j}$, we can now rewrite the perturbation series for the steady state in the form

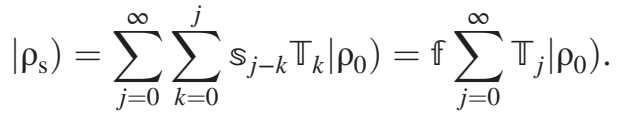

Here, the superoperator $\mathbb{f}=\mathbb{f}\left(\mathbb{S}_{1}, \mathbb{S}_{2}, \ldots\right)=\sum_{n=0}^{\infty} \mathbb{S}_{n}$ implements the resummation of terms that we have been aiming for. It is given by

$\mathbb{f}=\mathbb{1}+\mathbb{S}_{2}+\mathbb{S}_{3}+\mathbb{S}_{4}+\mathbb{S}_{2}^{2}+\mathbb{S}_{5}+\mathbb{S}_{2} \mathbb{S}_{3}+\mathbb{S}_{3} \mathbb{S}_{2} \ldots$,

i.e., the sum of all possible products of $\mathbb{S}$ superoperators (explicitly shown up to fifth order here). Diagrammatically, we represent $\mathbb{f}$ in the form shown in Fig. 3(b). Because of the definitions of $\mathbb{S}_{j}$ and $\mathbb{T}_{j}$ as diagonal and off-diagonal superoperators, $\mathbb{S}_{j}$ corresponds to a loop diagram with initial and final states being identical, all $(j-1)$ intermediate states being different from the initial or final state, and consecutive intermediate states being different from each other.

The role of $\mathbb{S}_{j}$ resembles that of an irreducible selfenergy contribution of order $j$ in closed-system perturbation theory. Similarly, the physical interpretation of $\mathbb{S}_{j}$ has to wait until we apply the formalism to a physical system, for example, the Jaynes-Cummings lattice model in Sec. IV C. Moreover, if we define $\mathbb{S}=\sum_{j=1}^{\infty} \mathbb{S}_{j}$ as the sum of all irreducible "self-energy" contributions, we can rewrite $\mathbb{f}=\sum_{j=0}^{\infty} \mathbb{S}^{j}=(\mathbb{1}-\mathbb{S})^{-1}$ and obtain

$$
\left.\left.\mid \rho_{\mathrm{s}}\right)=\sum_{j=0}^{\infty} \frac{1}{\mathbb{1}-\mathbb{S}} \mathbb{T}_{j} \mid \rho_{0}\right)
$$

for our resummed series expansion of the steady state. Because of the $(\mathbb{1}-\mathbb{S})^{-1}$ prefactor, each term $\left.\mid \rho^{[j]}\right)=$ $\left.(\mathbb{1}-\mathbb{S})^{-1} \mathbb{T}_{j} \mid \rho_{0}\right)$ in this sum includes perturbative corrections up to infinite order. We therefore call the term $\mid \rho^{[j]}$ ) the rank- $j$ term of the resummed series. We note that, formally, Eq. (31) is an exact expression for the steady state. Practical calculations will typically involve a truncation in both the maximum summation index $j$ and the maximum order of irreducible self-energy contributions taken into account.

We represent individual terms $\mid \rho^{[j]}$ ) in the resummed series by the type of diagram shown in Fig. 3(c). The finalstate dot is marked with a circle to indicate the inclusion of the self-energy correction. The diagrammatic rules are similar to the case without resummation, Fig. 3(a), except that the off-diagonal nature of $\mathbb{T}_{j}$ in addition requires that all intermediate states be different from the final state. 
This is simple to infer when writing $\mathbb{T}_{j}$ in the form of Eq. (22). Each diagram then translates to an expression with the following structure:

$$
\begin{aligned}
\left.\mid \rho^{[j]}\right)= & \left.\sum_{\mu_{j}} \sum_{\nu_{1}, \ldots, \nu_{j-1} \neq \mu_{j}} \mid \mathbf{u}_{\mu_{j}}^{0}\right)\left(\frac{1}{\mathbb{1}-\mathbb{S}}\right)_{\mu_{j} \mu_{j}} \\
& \times(\mathbb{U})_{\mu_{j} \nu_{j-1}}(\mathbb{U})_{\nu_{j-1} \nu_{j-2}} \ldots(\mathbb{U})_{\nu_{2} \nu_{1}}(\mathbb{U})_{\nu_{1} 0} .
\end{aligned}
$$

\section{APPLICATION TO THE OPEN JAYNES-CUMMINGS LATTICE}

Lindblad perturbation theory and resummation as discussed in the previous section are applicable to a large class of open quantum systems. Here, we present its concrete use in studying the open Jaynes-Cummings lattice [Eqs. (1) and (3)-(5)] as a specific example of an open driven quantum lattice. The example is of particular interest because of its role as a minimal model for highly anticipated experiments with circuit-QED lattices [64]. The photonic backbone of the lattice has already been demonstrated in the experimental work by Underwood et al. [51].

\section{A. Preparatory steps}

We consider a uniform lattice, in which resonator frequencies, qubit frequencies, and related quantities have uniform values across the lattice. (Disorder levels, especially in qubit frequencies, may need to be considered carefully for detailed modeling of future circuit-QED experiments, but this consideration is beyond the scope of the present paper.) We find the modes of the photonic lattice structure by diagonalizing the $N \times N$ hopping matrix, $N$ being the number of lattice sites [84]. For periodic lattices, diagonalization is achieved in the usual way by switching from real space to momentum space via the transformation $\mathrm{a}_{\mathbf{r}}=\sum_{\mathbf{k}} \tilde{a}_{\mathbf{k}} e^{i \mathbf{k} \cdot \mathbf{r}} / \sqrt{N}$. Here, photons inside the mode with quasimomentum $\mathbf{k}$ are annihilated by $\tilde{\mathbf{a}}_{\mathbf{k}}$, and $\mathbf{k}$ runs over all reciprocal lattice vectors from the first Brillouin zone. Note that $\mathbf{k}=\mathbf{0}$ corresponds to the uniform mode with identical amplitudes on all sites, which is the mode being excited by a global coherent drive.

Depending on the values of model parameters, it is beneficial to perform a displacement transformation that eliminates the coherent drive on the photon mode and converts it into an effective qubit drive instead. This is particularly helpful when the uniform photon mode is approximately in a coherent state with a large number of photons. The coherent displacement then serves as a tool incorporating this insight directly into the unperturbed Liouville superoperator and mitigates the need for large photon number cutoffs. (In a regime of low photon occupation, however, the displacement transformation can be skipped and the perturbative treatment carried out directly.) The displacement is applied to the $\mathbf{k}=\mathbf{0}$ mode, i.e., $\mathrm{b}_{\mathbf{0}}=\tilde{\mathrm{a}}_{\mathbf{0}}-\alpha$, with $\alpha=-\sqrt{N} \epsilon /\left(\delta \omega_{\mathbf{k}=\mathbf{0}}-i \gamma / 2\right)$.
After this displacement, the resonator drive is converted into an effective qubit drive of strength $\epsilon_{\mathrm{q}}=g \alpha / \sqrt{N}$. The resulting Hamiltonian has the form

$$
\mathrm{H}^{\prime}=\sum_{\mathbf{k}} \mathrm{H}_{r}^{\mathbf{k}}+\sum_{\mathbf{r}} \mathrm{H}_{q}^{\mathbf{r}}+\sum_{\mathbf{k}, \mathbf{r}} \mathrm{H}_{r q}^{\mathbf{k r}},
$$

where the three terms correspond to the photon, qubit, and photon-qubit coupling contributions. The resonator part now lacks the drive term, $\mathrm{H}_{r}^{\mathbf{k}}=\delta \omega_{\mathbf{k}} \mathrm{b}_{\mathbf{k}}^{\dagger} \mathrm{b}_{\mathbf{k}}$. (We define $\mathrm{b}_{\mathbf{k}}=\tilde{\mathrm{a}}_{\mathbf{k}}$ for $\mathbf{k} \neq \mathbf{0}$ to unify notation.) The qubit Hamiltonian including the effective drive reads

$$
\mathrm{H}_{q}^{\mathrm{r}}=\delta \Omega \sigma_{\mathbf{r}}^{+} \sigma_{\mathbf{r}}^{-}+\left(\epsilon_{\mathrm{q}} \sigma_{\mathbf{r}}^{+}+\epsilon_{\mathrm{q}}^{*} \sigma_{\mathbf{r}}^{-}\right),
$$

and the interaction Hamiltonian is given by

$$
\mathrm{H}_{r q}^{\mathbf{k r}}=\frac{g}{\sqrt{N}}\left(\mathrm{~b}_{\mathbf{k}} \sigma_{\mathbf{r}}^{+} e^{i \mathbf{k} \cdot \mathbf{r}}+\mathrm{b}_{\mathbf{k}}^{\dagger} \sigma_{\mathbf{r}}^{-} e^{-i \mathbf{k} \cdot \mathbf{r}}\right) .
$$

Finally, the dissipator term simply transforms as $\gamma \sum_{\mathbf{r}} \mathbb{D}\left[\tilde{\mathbf{a}}_{\mathbf{r}}\right]=\gamma \sum_{\mathbf{k}} \mathbb{D}\left[b_{\mathbf{k}}\right]$.

In the absence of the interaction $\mathrm{H}_{r q}^{\mathrm{kr}}$, resonator modes and qubits decouple, and the associated master equation is exactly solvable. This presents us with an ideal starting point for a perturbative treatment of $\mathrm{H}_{r q}^{\mathrm{kr}}$, which physically is a very sensible treatment of the dispersive regime. The unperturbed Liouville superoperator is then $\mathbb{L}_{0}=\sum_{\mathbf{k}} \mathbb{L}_{r}^{\mathbf{k}}+$ $\sum_{\mathbf{r}} \mathbb{L}_{q}^{\mathbf{r}}$ with separate photon contribution, $\mathbb{L}_{r}^{\mathbf{k}} \bullet=-i\left[\mathrm{H}_{r}^{\mathbf{k}}, \bullet\right]+$ $\mathbb{D}\left[\mathrm{b}_{\mathbf{k}}\right] \bullet$, and qubit contribution, $\mathbb{L}_{q}^{\mathbf{r}} \bullet-i\left[\mathrm{H}_{q}^{\mathbf{r}}, \bullet\right]+\mathbb{D}\left[\sigma_{\mathbf{r}}^{-}\right] \bullet$.

\section{B. Exact solution for the unperturbed lattice problem}

We can diagonalize the photonic part $\sum_{\mathbf{k}} \mathbb{L}_{r}^{\mathbf{k}}$ analytically by using the third-quantization method [65-72] (or alternative techniques; see, e.g., Ref. [85]). This method employs the superoperators $\mathbb{b}_{\mathbf{k}}, \mathfrak{b}_{\mathbf{k}}^{*}, \mathbb{C}_{\mathbf{k}}, \mathbb{C}_{\mathbf{k}}^{*}$, which mimic boson annihilation and creation operators and are defined by

$$
\begin{aligned}
& \mathfrak{b}_{\mathbf{k}} \rho=b_{\mathbf{k}} \rho \text { and } \quad \mathfrak{b}_{\mathbf{k}}^{\dagger} \rho=b_{\mathbf{k}}^{\dagger} \rho-\rho b_{\mathbf{k}}^{\dagger}, \\
& \mathbb{C}_{\mathbf{k}} \rho=\rho b_{\mathbf{k}}^{\dagger} \quad \text { and } \quad \mathbb{C}_{\mathbf{k}}^{\ddagger} \rho=\rho b_{\mathbf{k}}-b_{\mathbf{k}} \rho .
\end{aligned}
$$

While $b_{\mathrm{r}}^{\ddagger}$ and $\mathbb{b}_{\mathrm{r}}$ are not proper adjoints, the use of the unconventional " $\neq$ " symbol is motivated by the fact that it leads to commutation relations of the ordinary form,

$$
\left[\mathfrak{b}_{\mathbf{k}}, \mathbb{b}_{\mathbf{k}^{\prime}}^{\vdots}\right]=\left[\mathbb{C}_{\mathbf{k}}, \mathbb{C}_{\mathbf{k}^{\prime}}^{\vdots}\right]=\delta_{\mathbf{k k}^{\prime}},
$$

and all other commutators vanish. Thanks to this commutator algebra, $\mathbb{L}_{r}^{\mathbf{k}}$ takes on the compact form [68]

$$
\mathbb{L}_{r}^{\mathbf{k}}=t_{\mathbf{k}} \mathbb{b}_{\mathbf{k}}^{\ddagger} \mathbb{b}_{\mathbf{k}}+t_{\mathbf{k}}^{*} \mathbb{C}_{\mathbf{k}}^{\ddagger} \mathbb{C}_{\mathbf{k}}
$$


Here, $\mathbb{b}_{\mathbf{k}}\left(\mathbb{b}_{\mathbf{k}}^{\ddagger}\right)$ and $\mathbb{C}_{\mathbf{k}}\left(\mathbb{C}_{\mathbf{k}}^{\ddagger}\right)$ may be viewed as "normalmode" superoperators with complex-valued "mode frequencies" $t_{\mathbf{k}}=-i \delta \omega_{\mathbf{k}}-\gamma / 2$.

From this result, it is straightforward to read off eigenvalues and eigenstates of $\mathbb{L}_{r}^{\mathbf{k}}$, analogous to the way one finds eigenvalues and eigenstates of a noninteracting boson Hamiltonian. For a given $\mathbf{k}$ mode, the right and left "vacuum states" obey $\left.\left.\mathbb{b}_{\mathbf{k}} \mid \mathbf{r}_{00}^{\mathbf{k}}\right)=\mathbb{C}_{\mathbf{k}} \mid \mathbf{r}_{00}^{\mathbf{k}}\right)=0$ and $\left(\breve{r}_{00}^{\mathbf{k}} \mid \mathfrak{b}_{\mathbf{k}}^{\ddagger}=\left(\breve{r}_{00}^{\mathbf{k}} \mid \mathbb{C}_{\mathbf{k}}^{\ddagger}=0\right.\right.$. The right vacuum state is therefore the projector $\left|\mathbf{r}_{00}^{\mathbf{k}}\right\rangle=\left|0_{\mathbf{k}}\right\rangle\left\langle 0_{\mathbf{k}}\right|$ onto the pure state without any photons in mode k. One can show that the left vacuum states must always coincide with the identity operator, $\left(\breve{r}_{00}^{\mathbf{k}} \mid=1\right.$.

The "excited" eigenstates of $\mathbb{L}_{r}^{\mathbf{k}}$ are obtained by acting with the creation superoperators on the vacuum states. For given $\mathbf{k}$, this means

$$
\left.\left.\mid \mathbf{r}_{m n}^{\mathbf{k}}\right)=\frac{1}{\sqrt{m ! n !}}\left(\mathbb{b}_{\mathbf{k}}^{\ddagger}\right)^{m}\left(\mathbb{C}_{\mathbf{k}}^{\ddagger}\right)^{n} \mid \mathbf{r}_{00}^{\mathbf{k}}\right)
$$

and

$$
\left(\breve{\mathbf{r}}_{m n}^{\mathbf{k}} \mid=\frac{1}{\sqrt{m ! n !}}\left(\breve{\mathrm{r}}_{00}^{\mathbf{k}} \mid\left(\mathbb{b}_{\mathbf{k}}\right)^{m}\left(\mathbb{C}_{\mathbf{k}}\right)^{n}\right.\right.
$$

The corresponding eigenvalues are $\lambda_{m n}^{\mathbf{k}}=m t_{\mathbf{k}}+n t_{\mathbf{k}}^{*}$ $(m, n=0,1, \ldots)$. When forming the appropriate product states and summing eigenvalues over $\mathbf{k}$, we thus obtain the entire spectrum of the photonic part $\sum_{\mathbf{k}} \mathbb{L}_{r}^{\mathbf{k}}$.

Moreover, the qubit Liouvillian $\sum_{\mathbf{r}} \mathbb{L}_{q}^{\mathbf{r}}$ can be diagonalized exactly since it decomposes into a direct product of

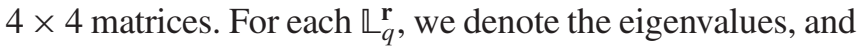
right and left eigenstates by $\left.\ell_{\mu}^{\mathbf{r}}, \mid \mathbf{q}_{\mu}^{\mathbf{r}}\right)$ and $\left(\breve{\mathbf{q}}_{\mu}^{\mathbf{r}} \mid\right.$, respectively $(\mu=0, \ldots, 3)$. Except for special parameters, analytical expressions for these quantities are too lengthy to provide much insight and will hence not be recorded here.

Altogether, right and left eigenstates of the full-lattice Liouvillian $\mathbb{L}_{0}$ thus take the form

$$
\left.\left.\left.\mid \mathbf{u}_{\vec{m} \vec{n} \vec{\mu}}^{0}\right)=\underset{\mathbf{k}}{\otimes} \mid \mathbf{r}_{m_{\mathbf{k}} n_{\mathbf{k}}}^{\mathbf{k}}\right) \underset{\mathbf{r}}{\otimes} \mid \mathbf{q}_{\mu_{\mathbf{r}}}^{\mathbf{r}}\right),
$$

with corresponding eigenvalues

$$
\lambda_{\vec{m} \vec{n} \vec{\mu}}^{0}=\sum_{\mathbf{k}} \lambda_{m_{\mathbf{k}} n_{\mathbf{k}}}^{\mathbf{k}}+\sum_{\mathbf{r}} \ell_{\mu_{\mathbf{r}}}^{\mathbf{r}} .
$$

The multi-indices $\vec{m}, \vec{n}$, and $\vec{\mu}$ collect the sets of all "quantum numbers" $m_{\mathbf{k}}, n_{\mathbf{k}}$, and $\mu_{\mathbf{r}}$. Thus, we are ready for the perturbative treatment of the Liouvillian $\mathbb{L}_{1}$ capturing the Jaynes-Cummings interaction $\mathrm{H}_{r q}^{\mathrm{kr}}$.

\section{Perturbative treatment and resummation}

The perturbation superoperator decomposes into a sum $\mathbb{L}_{1}=\sum_{\mathbf{k}, \mathbf{r}} \mathbb{L}_{1}^{\mathbf{k r}}$, in which each term describes the interaction between an individual resonator mode $\mathbf{k}$ and a qubit at position $\mathbf{r}$ :

$$
\mathbb{L}_{1}^{\mathbf{k r}} \bullet=-i \frac{g}{\sqrt{N}}\left[\mathrm{~b}_{\mathbf{k}} \sigma_{\mathbf{r}}^{+} e^{i \mathbf{k} \cdot \mathbf{r}}+\mathrm{b}_{\mathbf{k}}^{\dagger} \sigma_{\mathbf{r}}^{-} e^{-i \mathbf{k} \cdot \mathbf{r}}, \bullet\right] .
$$

It is therefore convenient to write the $\mathbb{U}$ superoperator (see Sec. III) as an analogous sum, i.e., $\mathbb{U}=\sum_{\mathbf{k}, \mathbf{r}} \mathbb{U}^{\mathbf{k r}}$ with $\mathbb{U}^{\mathbf{k r}}=-\mathbb{L}_{0}^{-1} \mathbb{L}_{1}^{\mathbf{k r}}$. Each $\mathbb{U}^{\mathbf{k r}}$ is off diagonal with respect to the unperturbed basis, so that $\mathbb{U}^{\mathbf{k r}}=\llbracket \mathbb{U}^{\mathbf{k r}} \rrbracket=\mathbb{T}_{1}^{\mathbf{k r}}$ holds.

The rank-1 term in the resummation [Eq. (31)] is given by

$$
\begin{aligned}
\left.\mid \rho^{[1]}\right) & \left.=\frac{1}{\mathbb{1}-\mathbb{S}} \sum_{\mathbf{k}, \mathbf{r}} \mathbb{U}^{\mathbf{k r}} \mid \rho_{0}\right) \\
& \left.=\sum_{\mathbf{k}, \mathbf{r}} \sum_{\vec{s}} \frac{1}{\mathbb{1}-\mathbb{S}} \mid \rho_{\mathbf{k} \mathbf{r} \vec{s}}^{[1]}\right)\left(\breve{\rho}_{\mathbf{k} \mathbf{r} \vec{s}}^{[1]}\left|\bigcup^{\mathbf{k r}}\right| \rho_{0}\right) .
\end{aligned}
$$

Here, $\left.\left.\left.\mid \rho_{\mathbf{k r} \vec{s}}^{[1]}\right)=\mid \mathbf{r}_{m n}^{\mathbf{k}} \mathbf{q}_{\mu}^{\mathbf{r}}\right) \underset{\mathbf{k}^{\prime} \neq \mathbf{k}}{\otimes} \mid \mathbf{k}_{00}^{\mathbf{k}^{\prime}}\right) \underset{\mathbf{r}^{\prime} \neq \mathbf{r}}{\otimes}\left|\mathbf{q}_{0}^{\mathbf{r}^{\prime}}\right\rangle$ is an interacting cluster involving the photon mode $\mathbf{k}$ and qubit at position $\mathbf{r}$ in state $\vec{s}=(m, n, \mu)$. In a similar manner, we see that the rank-2 term

$$
\begin{aligned}
& \left.\left.\mid \rho^{[2]}\right)=\frac{1}{\mathbb{1}-\mathbb{S}} \sum_{\mathbf{k}, \mathbf{k}^{\prime}, \mathbf{r}, \mathbf{r}^{\prime}} \llbracket \llbracket \mathbb{U}^{\mathbf{k r}} \rrbracket \mathbb{U}^{\mathbf{k}^{\prime} \mathbf{r}^{\prime}} \rrbracket \mid \rho_{0}\right) \\
& \left.=\sum_{\mathbf{k}, \mathbf{k}^{\prime}, \mathbf{r}, \mathbf{r}^{\prime}} \sum_{\left[\vec{s} \overrightarrow{s^{\prime}}\right]} \frac{1}{\mathbb{1}-\mathbb{S}} \mid \rho_{\mathbf{k r r s} ; \mathbf{k}^{\prime} \mathbf{r}^{\prime} \vec{s}^{\prime}}^{[2]}\right) \\
& \times\left(\breve{\rho}_{\mathbf{k} \mathbf{r} \vec{s} ; \mathbf{k}^{\prime} \mathbf{r}^{\prime} \bar{s}^{\prime}}^{[2]}\left|\mathbb{U}^{\mathbf{k r}}\right| \rho_{\mathbf{k}^{\prime} \mathbf{r}^{\prime} \bar{s}^{\prime}}^{[1]}\right)\left(\breve{\rho}_{\mathbf{k}^{\prime} \mathbf{r}^{\prime} s^{\prime}}^{[1]}\left|\mathbb{U}^{\mathbf{k}^{\prime} \mathbf{r}^{\prime}}\right| \rho_{0}\right)
\end{aligned}
$$

incorporates the resummation of a cluster $\left.\mid \rho_{\mathbf{k r} \vec{r}, \mathbf{k}^{\prime} \mathbf{r}^{\prime} s^{\prime}}^{[2]}\right)$ composed of two photon modes and two qubits in states $\vec{s}$ and $\overrightarrow{s^{\prime}}$. (The bracket notation in the corresponding summation signals that the allowed choices of these states is dictated by the off-diagonalism requirements in $\mathbb{T}_{2}=\llbracket\left[\mathbb{U}^{\mathbf{k r}} \rrbracket \mathbb{U}^{\mathbf{k}^{\prime} \mathbf{r}^{\prime}} \rrbracket\right.$.) In the definition of $\mid \rho_{\mathbf{k} \mathbf{r} \vec{s}, \mathbf{k}^{\prime} \mathbf{r}^{\prime} \underline{s}^{\prime}}^{[2]}$, several cases must be distinguished according to whether $\mathbf{k}=\mathbf{k}^{\prime}$ and/or $\mathbf{r}=\mathbf{r}^{\prime}$. For the case where both pairs are distinct, we have

$$
\left.\left.\left.\mid \rho_{\mathbf{k} \mathbf{r}, \mathbf{k}^{\prime} \mathbf{r}^{\prime} \underline{s}^{\prime}}^{[2]}\right)=\left|\mathbf{r}_{m n}^{\mathbf{k}} \mathbf{r}_{m^{\prime} n^{\prime}}^{\mathbf{k}^{\prime}} \mathbf{q}_{\mu}^{\mathbf{r}} q_{\mu^{\prime}}^{\left.\mathbf{r}^{\prime}\right)} \underset{\mathbf{k}^{\prime \prime} \neq \mathbf{k}, \mathbf{k}^{\prime}}{\otimes}\right| r_{00}^{\mathbf{k}^{\prime \prime}}\right) \underset{\mathbf{r}^{\prime \prime} \neq \mathbf{r}, \mathbf{r}^{\prime}}{\otimes} \mid q_{0}^{\mathbf{r}^{\prime \prime}}\right) .
$$

Analogous definitions hold in the other three cases.

By inspection of Eqs. (45) and (46), we expect that, in general, the rank- $j$ correction consists of a sum over all possible terms in which clusters of $j$ photon modes and $j$ qubits deviate from the unperturbed density matrix. Thanks to resummation, interaction within each cluster includes terms up to infinite order. We note that this cluster structure directly implies a hierarchy of correlations with increasing rank $j$. Specifically, each $n$-point correlation function with $p$ photon and $q$ qubit operators, $\left\langle\mathrm{b}_{\mathbf{k}_{1}}^{(\dagger)} \cdots \mathrm{b}_{\mathbf{k}_{p}}^{(\dagger)} \sigma_{\mathbf{r}_{1}}^{a_{1}} \cdots \sigma_{\mathbf{r}_{q}}^{a_{q}}\right\rangle_{\mathrm{ss}}$, 
does not trivially separate into a product of correlators if the rank $j$ of the correction satisfies $j \geq \max \{p, q\}$. We also emphasize that clusters automatically include long-range correlations between distant qubits.

The essential tasks of determining the perturbative corrections and resummation consist of evaluating matrix elements of the form given in Eqs. (45) and (46) and computing the effect of the resummation superoperator $\mathbb{S}$ to a given order. We illustrate the procedure for the example of rank-1 corrections. Plugging in the definition of $\mathbb{U}^{\mathbf{k r}}$ and recalling that $\mathbb{L}_{0}$ is diagonal with respect to the unperturbed basis states, we obtain

$$
\begin{aligned}
\left(\breve{\rho}_{\mathbf{k r} \vec{s}}^{[1]}\left|\mathbb{U}^{\mathbf{k r}}\right| \mathbf{r}_{00}^{\mathbf{k}} \mathbf{q}_{0}^{\mathbf{r}}\right) & =-\left(\breve{r}_{m n}^{\mathbf{k}} \breve{\mathbf{q}}_{\mu}^{\mathbf{r}}\left|\mathbb{L}_{1}^{\mathbf{k r}}\right| \mathbf{r}_{00}^{\mathbf{k}} \mathbf{q}_{0}^{\mathbf{r}}\right) / \lambda_{\mathbf{k r} \vec{s}} \\
& =i \operatorname{tr}\left(\breve{\mathbf{r}}_{m n}^{\breve{k}^{\dagger} \dagger} \breve{\mathbf{q}}_{\mu}^{\mathbf{r} \dagger}\left[\mathrm{H}_{r q}^{\mathbf{k r}}, \mathbf{r}_{00}^{\mathbf{k}} \mathbf{q}_{0}^{\mathbf{r}}\right]\right) / \lambda_{\mathbf{k r} \vec{s}},
\end{aligned}
$$

where $\lambda_{\mathbf{k r} \vec{s}}=\lambda_{m n}^{\mathbf{k}}+\ell_{\mu}^{\mathbf{r}}$. Once the commutator is opened, it is useful to note that the simple properties of the $\mathbb{L}_{r}$ eigenstates lead to vanishing overlaps $\operatorname{tr}\left(\breve{\mathbf{r}}_{m n}^{\mathbf{k}} \mathbf{b}_{\mathbf{k}}^{(\dagger)} \mathbf{r}_{m n}^{\mathbf{k}}\right)=$ $\operatorname{tr}\left(\breve{\mathbf{r}}_{m n}^{\mathbf{k} \dagger} \mathbf{r}_{m n}^{\mathbf{k}} \mathbf{b}_{\mathbf{k}}^{(\dagger)}\right)=0$, so any application of $\mathbb{U}^{\mathbf{k r}}$ must switch to a different resonator-mode eigenstate. The same does not hold for traces of the qubit degrees of freedom; i.e., the overlaps $\operatorname{tr}\left(\breve{\mathbf{q}}_{\mu}^{\mathbf{k} \dagger} \sigma_{\mathbf{r}}^{+} \mathbf{q}_{\mu}^{\mathbf{k}}\right)$, etc., may indeed be nonzero. As a result, we obtain the two types of terms for the rank-1 correction, which are diagrammatically depicted in Fig. 4(a). The evaluation of rank-2 corrections follows the same basic scheme. Unsurprisingly, it is more tedious, and we only show two examples of corresponding diagrams in Fig. 4(b).

The effect of the resummation superoperator is to redistribute weights among cluster contributions. Since $\mathbb{S}$ is diagonal with respect to unperturbed Liouvillian eigenstates, we can cast its contribution into a particular form $\left.\left.\mathbb{S} \mid \mathbf{u}_{\vec{s}}^{0}\right)=\mid \mathbf{u}_{\vec{s}}^{0}\right)\left(\breve{u}_{\vec{s}}^{0}|\mathbb{S}| u_{\vec{s}}^{0}\right)$ and evaluate the occurring matrix element as follows. We choose an appropriate truncation for the series $\mathbb{S}=\mathbb{S}_{2}+\mathbb{S}_{3}+\cdots$ of irreducible resummation operators $\left(\mathbb{S}_{j}\right)_{\vec{s} \vec{s}^{\prime}}=\delta_{\vec{s} \vec{s}^{\prime}}\left(\mathbb{T}_{j-1} \mathbb{U}\right)_{\vec{s} \vec{s}^{s^{\prime}}}$. Matrix elements for $\mathbb{S}_{j}$ are calculated in the same way as for $\mid \rho^{[j]}$ ) except that the final state of the chain must be identical to the initial state. Figure 5 shows the resulting two diagrams for $\mathbb{S}_{2}$. Physically, these diagrams and their resummation capture the fact that the coupling between resonator modes and qubits alters the frequencies and lifetimes of Liouvillian eigenstates. Specifically, the resummation accounts for virtual processes in which excitations are repeatedly created, exchanged between qubits and resonator modes, and annihilated. The first diagram in Fig. 5, for instance, describes the creation of a qubit excitation, its swapping into a resonator mode and back into a qubit, and final annihilation. (The analogous reverse process where a missing excitation is swapped back and forth is included in this diagram as well.) The second diagram differs from the former by the altered qubit state in the virtual intermediate state. These second-order diagrams involve at most one excitation at a time; higher-order virtual processes allow more excitations. We emphasize that the physical interpretation is based on eigenstates of the Liouvillian rather than states in Hilbert space.

The above calculation of perturbative corrections and resummation may be simplified if we are merely interested in steady-state expectation values (rather than in the density matrix itself). As an example, consider computing an expectation value of a local qubit operator $\sigma_{\mathbf{r}}^{a}$ up to corrections of rank $j,\left\langle\sigma_{\mathbf{r}}^{a}\right\rangle \approx \sum_{j^{\prime}=0}^{j} \operatorname{tr}\left(\sigma_{\mathbf{r}}^{a} \rho^{\left[j^{\prime}\right]}\right)$. To effect the desired simplification, we recall that all eigenstates of $\mathbb{L}_{r}^{\mathbf{k}}$ and $\mathbb{L}_{q}^{\mathbf{r}}$ other than the steady state must be traceless, i.e., $\operatorname{tr}\left(\mathbf{q}_{\mu}^{\mathbf{r}}\right)=0$ for $\mu \neq 0$ and $\operatorname{tr}\left(\mathbf{r}_{m n}^{\mathbf{k}}\right)=0$ for nonzero $m$ or $n$ [86]. Therefore, any perturbative contribution $\sim \operatorname{tr}\left(\sigma_{\mathbf{r}}^{a} \mathbf{u}_{\vec{m} \vec{n} \vec{\mu}}^{0}\right)$ in which $\mu_{\mathbf{r}^{\prime}} \neq 0$ for some $\mathbf{r}^{\prime} \neq \mathbf{r}$ will immediately vanish since the partial trace over the qubit at position $\mathbf{r}^{\prime}$ is zero. Similarly, any term with $\left(m_{\mathbf{k}}, n_{\mathbf{k}}\right) \neq(0,0)$ for some photon mode $\mathbf{k}$ will vanish. As a result, none of the rank-1 corrections [Fig. 4(a)] contributes to local qubit expectation values. Only those diagrams that terminate in a state labeled (r) will yield a nonzero contribution to $\left\langle\sigma_{\mathbf{r}}^{a}\right\rangle$. Analogous diagrammatic rules apply for photon-mode operators. (a)

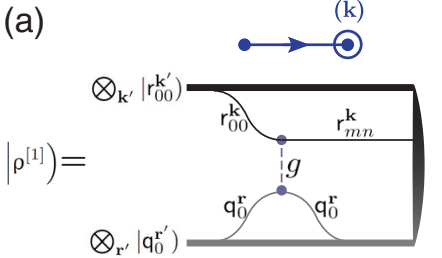

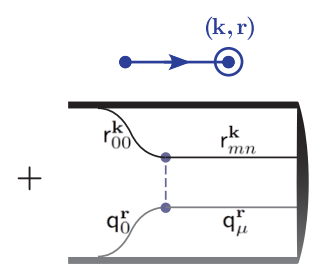

(b)

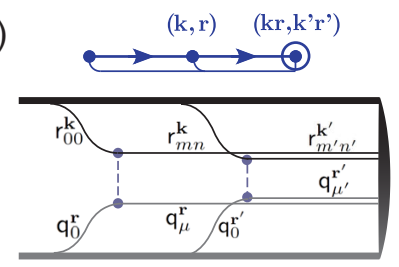

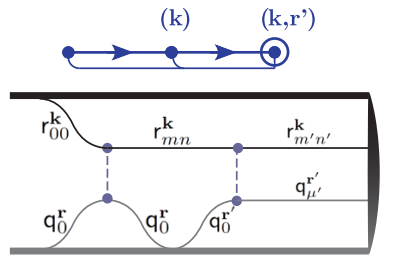

FIG. 4. Diagrams for perturbative treatment and resummation of a JC lattice. In each panel, the top part shows the general diagram labeled by constituents deviating from the steady-state configuration. The bottom parts are JC-lattice specific diagrams, with the upper branch denoting photon modes and the lower one qubit degrees of freedom. (a) Rank-1 corrections. There are two classes of terms with either a photon mode or a cluster of a photon mode $\mathbf{k}$ and a qubit at $\mathbf{r}$ deviating from the unperturbed steady-state configuration. Each interaction vertex $g$ must switch the photon mode configuration but may leave that of the qubit unchanged. Terminating symbols on the right signal application of the resummation superoperator $(\mathbb{1}-\mathbb{S})^{-1}$. (b) Two examples of rank-2 corrections, which differ in the number of involved photon modes and qubits and in the number of constituents deviating from the steady-state configuration. 


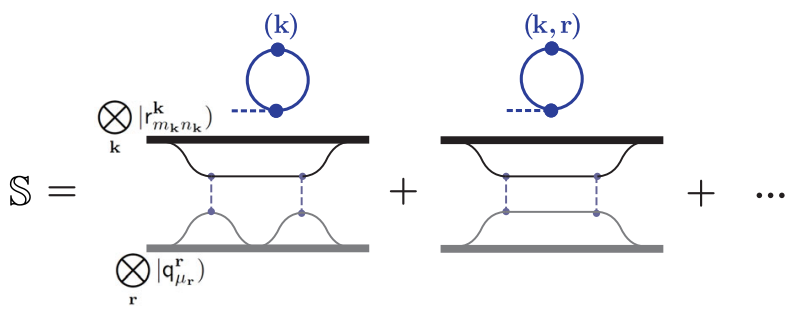

FIG. 5. Evaluation of the $\mathbb{S}$ superoperator. $\mathbb{S}$ is needed for resummation, and it is composed of irreducible diagrams starting and terminating in the same state. The two diagrams show the leading-order contributions, $\mathbb{S}_{2}$.

\section{Perturbative results for a near-resonant regime}

We now illustrate the validity and improvement achieved by (partial) resummation of the Lindblad perturbation series. For this purpose, we compare perturbative results for finite-size Jaynes-Cummings chains [Fig. 2(a)] to exact results computed via quantum trajectories methods. Perturbative resummation further allows us to treat periodic chains (or, open chains if desired) of sizes beyond the computational capabilities of exact quantum-trajectory solutions. Finally, we can carry out perturbative resummation even for an infinite system with chain or globalcoupling geometry. This versatility enables us to predict finite-size effects and the approach to the thermodynamic limit, as well as differences according to distinct lattice geometries. (We discuss the quite moderate computational costs of the perturbative treatment in Appendix B.)

In our treatment, we capture photon-mediated qubitqubit interactions by second-order Lindblad perturbation theory with resummation based on single-loop terms $\mathbb{S}_{2}$ (i.e., corrections of rank 2 in the above terminology). The most natural regime for treating the Jaynes-Cummings coupling perturbatively in this way is the dispersive regime where the detuning $\Delta=\min _{\mathbf{k}}\left|\Omega-\omega_{\mathbf{k}}\right|$ between qubit and photon-mode frequencies is large compared to their mutual coupling strength $g$ [87]. We have confirmed by exact numerics that the perturbation theory is indeed reliable in this regime and, over a wide range of parameters, we identify $g^{2} / \Gamma \Delta$ as the relevant small parameter governing the series expansion.

In the following, we choose to present results from exploring a different parameter regime more directly based on the open-system nature of Jaynes-Cummings lattices. Specifically, we consider the case where photon hopping dominates over both photon decay and Jaynes-Cummings coupling and where the latter two are permitted to be of the same order, i.e., $\kappa \gg g \sim \gamma$. The strong hopping $\kappa$ shifts the spectral weight of the photon modes away from the bare resonator frequency, which is chosen to be degenerate with the qubit frequency, $\Omega=\omega$. This regime is not fully dispersive, so nonlinearities are more pronounced and the significance of resummation becomes easily visible. Moreover, the condition $g \sim \gamma$ results in weakly perturbed coherent states for the photon modes over the full range of weak to strong drive strengths, without any particular limitation.

We begin with the comparison between perturbative and exact results for the steady state of few-site JaynesCummings chains with periodic boundary conditions. In our calculations, we have considered several qubit and resonator expectation values. Among those, we find that $\left|\left\langle\sigma^{-}\right\rangle\right|=\sqrt{\left\langle\sigma^{x}\right\rangle^{2}+\left\langle\sigma^{y}\right\rangle^{2}} / 2$ is a convenient choice for clearly resolving resonances. Representing the reduced steady-state density matrix for one of the qubits by means of the Bloch sphere picture, this quantity is directly proportional to the distance of the Bloch vector from the $z$ axis [see Fig. 6(a)]. Computing exact steady-state solutions for Jaynes-Cummings chains even as small as
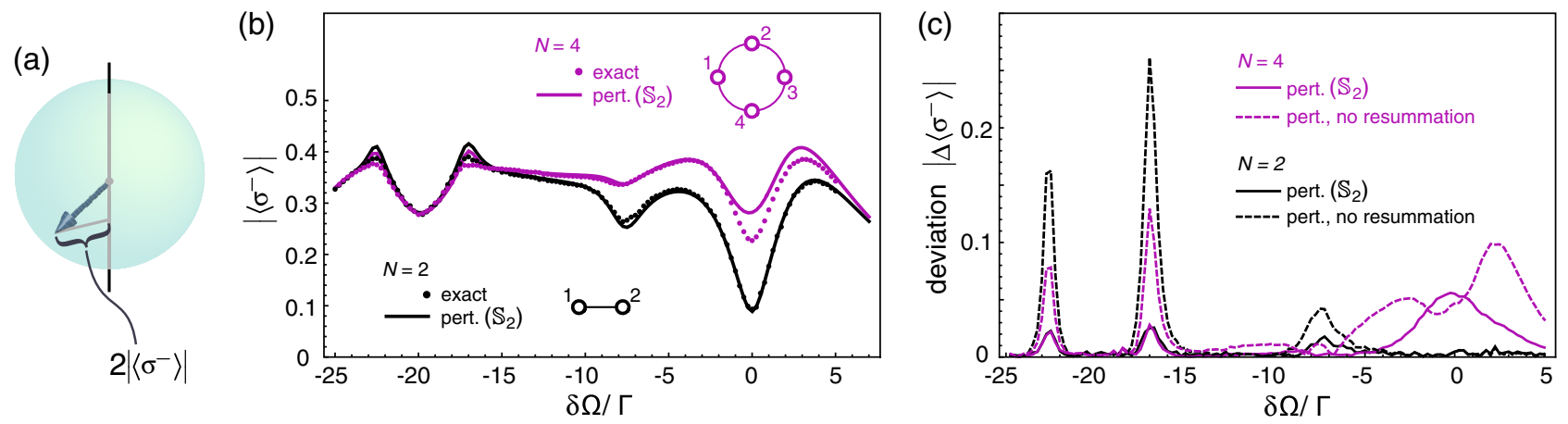

FIG. 6. Comparison between perturbative results and exact solution. (a) Within the Bloch-sphere picture, the steady-state expectation value $\left|\left\langle\sigma^{-}\right\rangle\right|$is directly proportional to the distance of the Bloch vector (representing $\rho_{\mathrm{s}}^{\text {qubit }}$ ) from the $z$ axis. Panel (b) shows $\left|\left\langle\sigma^{-}\right\rangle\right|$as a function of detuning $\delta \Omega$ between drive and bare qubit frequency, in units of the qubit relaxation rate $\Gamma$. Qubits are held in resonance with the bare resonator frequency, $\Omega=\omega$. Exact and perturbative results for chain sizes $N=2$ and 4 are in good agreement. (See text for explanation of deviations close to $\delta \Omega=0$ in the $N=4$ case.) (c) Curves here depict the absolute deviations between exact and perturbative results for calculations with (solid lines) and without resummation (dashed lines). Perturbation theory is not sufficient to describe the region close to $\delta \Omega=0$ for $N=4$ (see text). Outside this region, resummation consistently improves agreement with the exact solution. (Parameters: $g / \Gamma=3, \epsilon / \Gamma=20, \kappa / \Gamma=10$, and $\gamma / \Gamma=4$.) 
four sites is a nontrivial task, which we accomplish by averaging of quantum trajectories. For instance, exact results presented in Fig. 6 for $N=4$ were determined from stochastic time evolution of a quantum state vector of size $10^{4}$. Sufficient averaging of a single data point required a runtime of several days on one core.

The comparison between exact and rank-2 perturbative data (including resummation at the level of $\mathbb{S}_{2}$ ) in Fig. 6(b) shows very good agreement and indicates that the resummation procedure closely matches the exact solution. Plots in this figure show the steady-state value of $\left|\left\langle\sigma^{-}\right\rangle\right|$as a function of the detuning $\delta \Omega=\Omega-\omega_{d}$ between the drive and the bare qubit frequency. Multiple resonances are visible over the chosen frequency range (the nature of which we will further discuss below). The only notable quantitative deviations occur for $N=4$ in the vicinity of the bare qubit frequency where $\delta \Omega=0$. This deviation has a simple explanation: A look ahead at Fig. 8(b) shows that the four-site chain has a photon mode with large spectral weight directly on resonance with the bare qubit frequency, so we must expect the perturbative treatment in $g$ to break down. With the exception of this finding, we conclude that resummation of the perturbative series in the chosen parameter regime works very well. The improvement gained over the pure second-order approximation is illustrated in Fig. 6(c). In this panel, curves show the difference between approximate and exact results, $\Delta\left\langle\sigma^{-}\right\rangle=$ $\left\langle\sigma^{-}\right\rangle_{\text {approx }}-\left\langle\sigma^{-}\right\rangle_{\text {exact }}$, for the case including resummation (solid lines) and lacking resummation (dashed lines). Excluding the pathological region for $N=4$ around $\delta \Omega=0$, we observe that resummation consistently improves the results, reducing the deviation from the exact solution. The improvement is especially significant in the resonance region between $\delta \Omega / \Gamma \approx-17$ and -23 . Here, the drive populates the uniform mode (centered at $\delta \Omega / \Gamma=-20)$ and renders photon-mediated qubit-qubit interaction important, making resummation of corrections up to infinite order particularly fruitful.

The improvement gained by including resummation is even more striking for the three-site chain. In Fig. 7, the comparison between exact and perturbative results (no resummation) shows three regions with significant deviations. The deviations for $\delta \Omega / \Gamma \approx-24$ and -14 arise from spurious resonance peaks, which we can trace back to the poles of the inverse $\mathbb{Z}_{0}^{-1}$ of the unperturbed Liouville superoperator [Eq. (12)]. Resummation nicely cures these spurious peaks, effectively by shifting away poles of the relevant superoperators. This indicates that including resummation has an effect beyond simply truncating the perturbative series at higher orders. The deviation for $\delta \Omega / \Gamma \approx 1$ is associated with the resonance dip being wrongly shifted. Resummation improves the prediction of not just the resonance position but also its shape. These resonances contain information on many-body effects due to the coupling between the resonators and qubits. Hence,

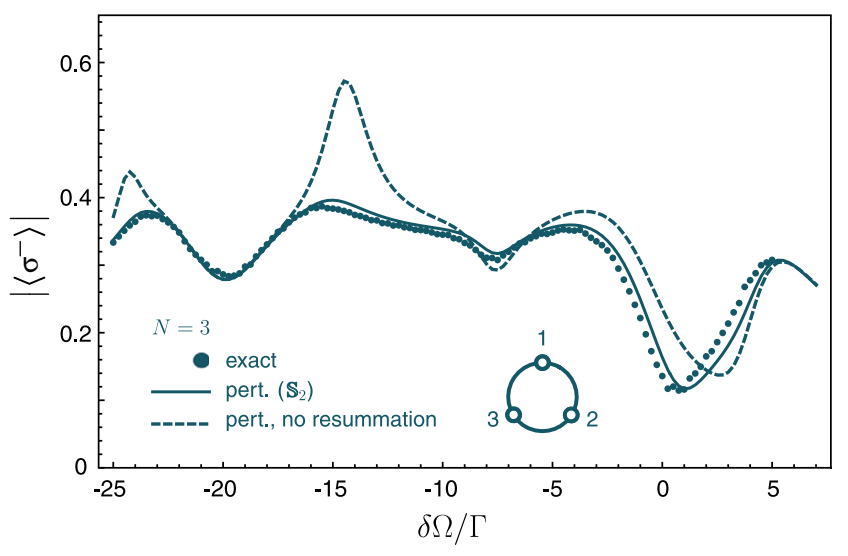

FIG. 7. Comparison between perturbative results with resummation (solid line) and without (dashed line) for the three-site chain. The result without resummation shows three regions with significant deviations from the exact solution (dots) for $\delta \Omega / \Gamma \approx-24,-14$ and 1 . These deviations (spurious and wrongly shifted resonances) are cured by including corrections obtained by resummation. (All system parameters are identical to those in Fig. 6.)

curing both wrongly shifted and spurious resonances by including resummation is vital and crucial for verification of experimental data.

We next turn to the discussion of the resonances visible in Fig. 6 and shown for additional site numbers $N$ in Fig. 8(a). For small chain lengths up to $N=5$ sites, we observe three resonances labeled $\mathrm{A}, \mathrm{B}$, and $\mathrm{C}$ in the range of drive frequencies spanning the photon-chain eigenmodes and qubit frequencies. [The uniform photon mode has the lowest frequency, $\delta \Omega / \Gamma=-20$, and the bare qubit frequency is at $\delta \Omega / \Gamma=0$; see vertical lines in Fig. 8(b)]. We find that the detailed positions and strengths of resonances depends on the number of sites, revealing systematic finitesize effects for chains of short lengths. Both the nature and $N$ dependence of resonances can be explained, or at least motivated, by the following considerations.

The resonance marked $A$ directly coincides with the frequency of the uniform photon mode. Equivalent interpretations of the resonance can be given based on the original Hamiltonian Eq. (3) with a coherent tone driving this particular mode with strength $\epsilon$, or for the Hamiltonian following the displacement transformation Eqs. (33) and (34). Employing the language of the latter description, we note that the strength $\epsilon_{\mathrm{q}}=-g \epsilon /\left(\delta \omega_{\mathbf{k}=\mathbf{0}}-i \gamma / 2\right)$ of the effective qubit drive reaches its local maximum at the uniform-mode frequency $(\delta \Omega / \Gamma=-20)$. This peak in the off-resonant Rabi drive, modified by weak coupling to photon modes, is responsible for resonance A. Its dependence on the site number $N$ is relatively weak and mainly affects the shoulders of the resonance. This is further confirmed by our results for the infinite-system case with periodic-chain and global-coupling geometry [Fig. 8(c)]. 

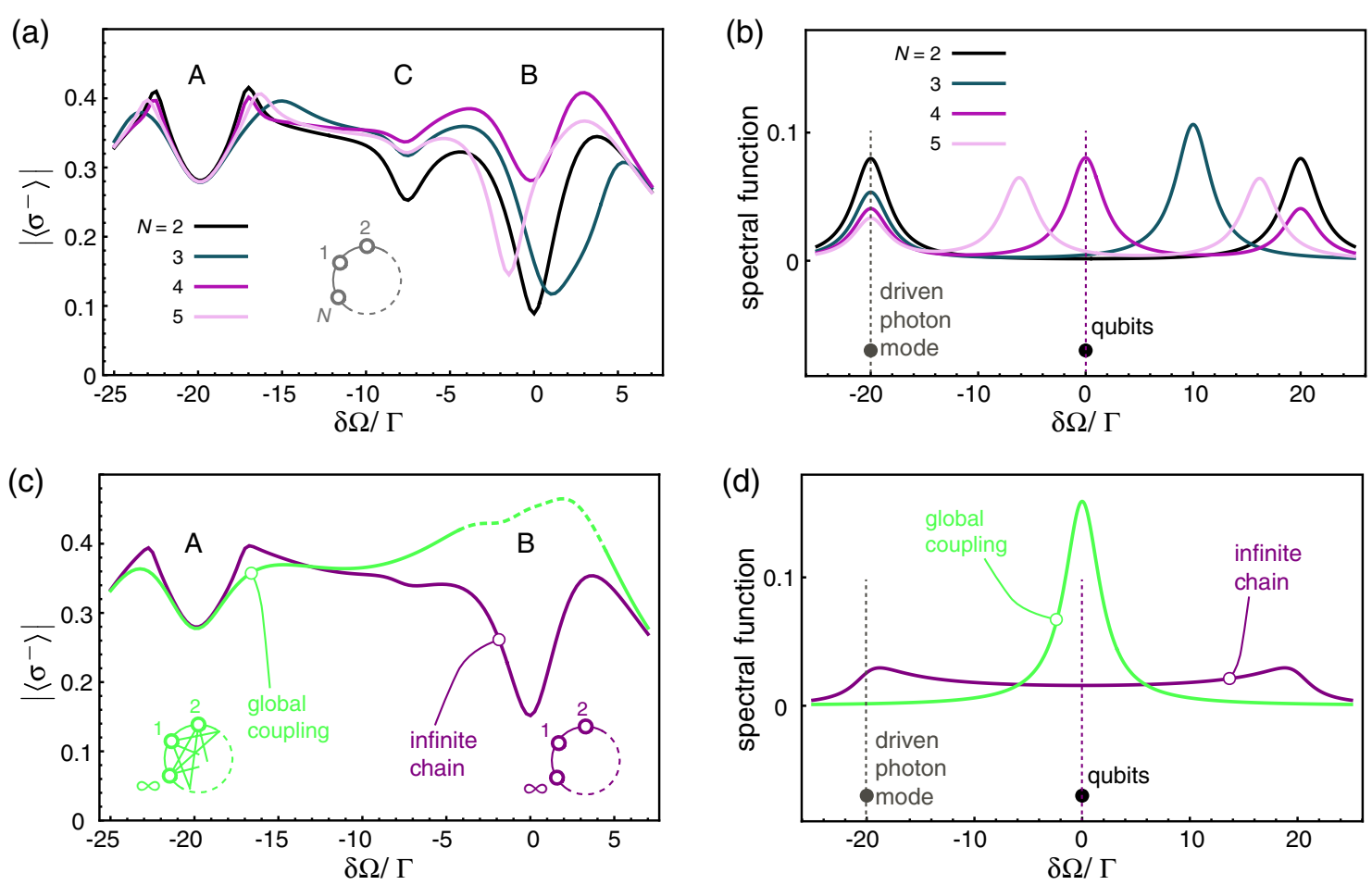

FIG. 8. Perturbative results and spectral functions for periodic Jaynes-Cummings chains and global-coupling model. (a) shows the qubit expectation value $\left|\left\langle\sigma^{-}\right\rangle\right|$for periodic chains with different site numbers $N$. We observe three resonance dips (labeled A-C). A and $\mathrm{B}$ are located roughly at the driven photon mode and qubit frequencies, respectively. (b) Spectral functions resonator modes differ for small site numbers. Presence and position of resonances near the qubit frequency explain the $N$-dependence of strength and shift of the B resonance. (c,d) show analogous plots for infinite lattices with periodic chain or global-coupling geometry. Resonances A and B are visible, but resonance $\mathrm{C}$ is (nearly) absent. [All system parameters are identical to those in Fig. 6.]

Both show the same resonance $A$ but differ in the resonance shoulders.

For resonance $\mathrm{B}$, the $N$ dependence of resonance position and strength is much more pronounced. The general region where $\mathrm{B}$ occurs is close to $\delta \Omega=0$, i.e., where bare qubit frequency and drive frequency match. Upon displacement, the drive transforms into an effective Rabi drive on each qubit [Eq. (34)]. Hence, the presence of a resonance is natural, and variations in its strength and precise position must be governed by the JaynesCummings interaction playing the role of the perturbation in our treatment [Eq. (44)]. The importance of this interaction is influenced by the position of photon-mode resonances $\omega_{\mathbf{k}}$ relative to the bare qubit frequency. In Figs. 8(b) and 8(d), we depict width and position of photon modes in terms of the spectral function $s(\omega)=\sum_{\mathbf{k}}\left[(\gamma / 2) /\left(\omega-\omega_{\mathbf{k}}\right)^{2}+(\gamma / 2)^{2}\right]$, which is the sum over individual Lorentzians for each photon mode, normalized such that $\int d \omega s(\omega)=1$. Inspection of resonance-B positions [Figs. 8(a) and 8(c)] and peaks in the spectral function [Figs. 8(b) and 8(d)] shows that peaks in $s(\omega)$ with significant weight in the region $\Omega \pm g$, shift B resonances towards the close-by photon mode (such as for $N=3,5$ ). Further, it is clear that strongly increased weight of the spectral function directly at the qubit frequency (such as for $N=4$ and for the global-coupling geometry) endangers the validity of perturbation theory in the JaynesCummings coupling. Above, we recognized this as the reason for the observed deviations between perturbation theory and an exact solution close to $\delta \Omega=0$ in the $N=4$ case. A look at the spectral function for the global-coupling geometry shows that the same issue occurs here. Accordingly, we show the perturbative result in Fig. 8(c) only with dashes in that region.

We note that steady-state expectation values for infinite lattices are not always easily accessible by other methods. Thanks to the possibility of carrying out leading-order resummation analytically in the infinite-system case, our treatment gives direct access to the thermodynamic limit of different lattice geometries. Here, we have chosen two extreme cases: the infinite periodic chain with a minimum number of links between sites and the global-coupling model with a maximum number of links. Figure 8(c) depicts results for both lattice structures. We expect that the region close to $\delta \Omega=0$ is unproblematic for the infinite chain case but potentially pathological for the globalcoupling model, which accumulates maximum spectral weight at the bare qubit frequency [Fig. 8(d)]. Away from the $\delta \Omega=0$ range, the two geometries yield similar behavior of $\left|\left\langle\sigma^{-}\right\rangle\right|$vs drive detuning $\delta \Omega$. As before, characteristic 

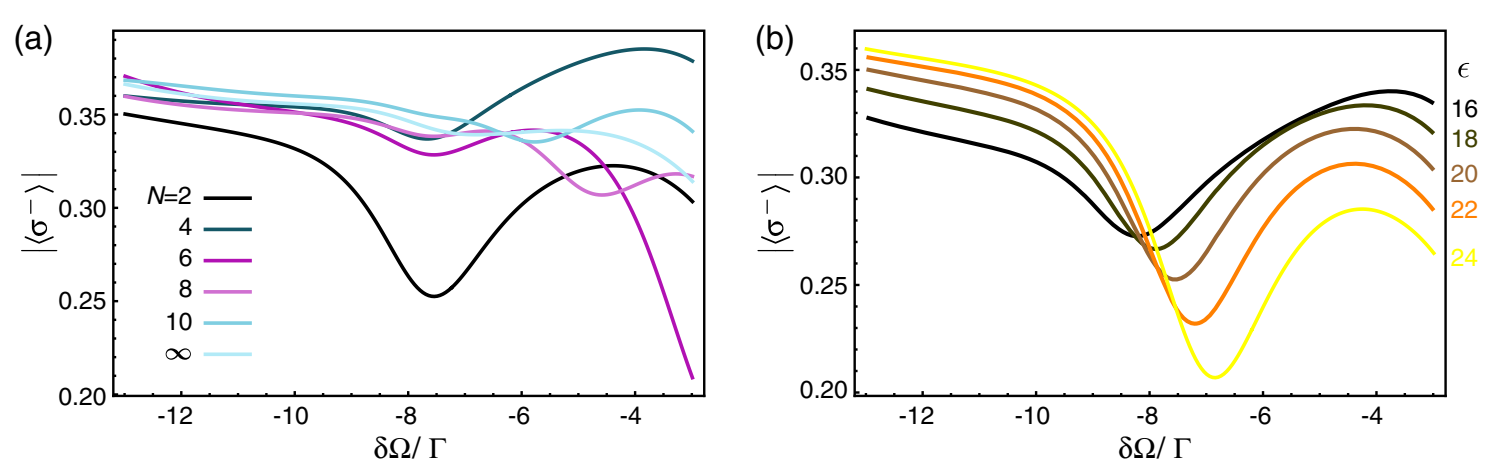

FIG. 9. Dependence of the anomalous resonance $\mathrm{C}$ on site number $N$ and drive strength $\epsilon$. (a) The anomalous dip becomes less prominent with increasing $N$ (even) and nearly vanishes for the infinite chain. (The same trend applies to odd site numbers.) (b) For the dimer case, $N=2$, the position of the anomalous resonance shifts monotonically with increasing drive strength $\epsilon$. The same trend is observed for longer chains. (Parameters are chosen the same as in Fig. 6.)

differences occur primarily in the shoulders of resonance $A$. Interestingly, resonance $\mathrm{C}$ is absent for both infinite lattices, and we discuss the rather anomalous behavior of this finite-size resonance next.

The anomalous properties of resonance $\mathrm{C}$ are illustrated in Fig. 9. The position of this resonance close to $\delta \Omega / \Gamma \approx 8$ does not simply coincide with a resonance between photon modes and bare qubits. Panel (a) shows the decrease of the resonance strength for increasing chain length $N$. Moreover, both resonance position and strength depend sensitively on the drive power, approximately $\epsilon$, as shown in Fig. 9 for the dimer case, $N=2$. We investigate this anomaly for the $N=2$ case, where a semiquantitative reduced model can shed light on the origin and nature of this resonance.

For $N=2$, we can confirm analytically that the anomalous resonance $\mathrm{C}$ is closely related to an eigenstates $|\psi\rangle$ of the displaced Hamiltonian $\mathrm{H}^{\prime}$ [Eq. (33)] without the effective drive. This eigenstate comprises two excitations distributed between the uniform mode and the qubits. Truncation to first order in $g / \kappa$ (a small quantity for the chosen parameter set) yields

$$
\begin{aligned}
|\psi\rangle \approx & \frac{1}{\sqrt{2}}\left|1_{\mathbf{0}}\right\rangle(|\uparrow \downarrow\rangle+|\downarrow \uparrow\rangle) \\
& -\frac{1}{\sqrt{2}} \frac{g}{\kappa}\left|2_{\mathbf{0}}\right\rangle|\downarrow \downarrow\rangle+\frac{1}{2} \frac{g}{\kappa}\left|0_{\mathbf{0}}\right\rangle|\uparrow \uparrow\rangle
\end{aligned}
$$

with eigenenergy $2 \Omega+2 \kappa-g^{2} / 2 \kappa$. Here, $\left|n_{\mathbf{0}}\right\rangle$ is the $n$-photon state of the uniform mode and $|\uparrow \uparrow\rangle$, etc., denote states of the qubits on the two sites. The effective drive Hamiltonian with strength $\epsilon_{\mathrm{q}}$ connects the ground state $|g\rangle=\left|0_{0}\right\rangle|\downarrow \downarrow\rangle$ to the state $|\psi\rangle$ via two intermediate states $|r\rangle$ and $|q\rangle$ (see Fig. 10). These intermediate states belong to the one-excitation manifold and primarily consist either of a photon in the uniform mode or of a qubit excitation, respectively. Truncated again to first order in $g / \kappa,|r\rangle$ and $|q\rangle$ are given by

$$
\begin{aligned}
& |r\rangle \approx\left|1_{\mathbf{0}}\right\rangle|\downarrow \downarrow\rangle+\frac{1}{2 \sqrt{2}} \frac{g}{\kappa}\left|0_{\mathbf{0}}\right\rangle(|\uparrow \downarrow\rangle+|\downarrow \uparrow\rangle), \\
& |q\rangle \approx \frac{1}{\sqrt{2}}\left|0_{\mathbf{0}}\right\rangle(|\uparrow \downarrow\rangle+|\downarrow \uparrow\rangle)-\frac{1}{2} \frac{g}{\kappa}\left|1_{\mathbf{0}}\right\rangle|\downarrow \downarrow\rangle .
\end{aligned}
$$

Our description of the anomalous resonance in the following is based on the effective four-level model spanned by the states $|g\rangle,|r\rangle,|q\rangle$, and $|\psi\rangle$ (see Fig. 10).

Within this model, the effective drive Hamiltonian connects the ground state $|g\rangle$ to the two-excitation state $|\psi\rangle$ via $|r\rangle$ and $|q\rangle$. Since the effective drive creates (or annihilates) qubit excitations only, there is stronger hybridization of $|g\rangle$ with $|q\rangle$ (strength of approximately $\epsilon_{\mathrm{q}}$ ) than with $|r\rangle$ (strength of approximately $\epsilon_{\mathrm{q}} g / \kappa$ ). An analogous argument applies to explain hybridization of $|r\rangle$ with $|\psi\rangle$ [again, strength of approximately $\epsilon_{\mathrm{q}}$ )]. We thus obtain the picture of two pairs of hybridized states, $|g\rangle \leftrightarrow|q\rangle$ and $|r\rangle \leftrightarrow|\psi\rangle$, with only small-drive matrix elements connecting the pairs.

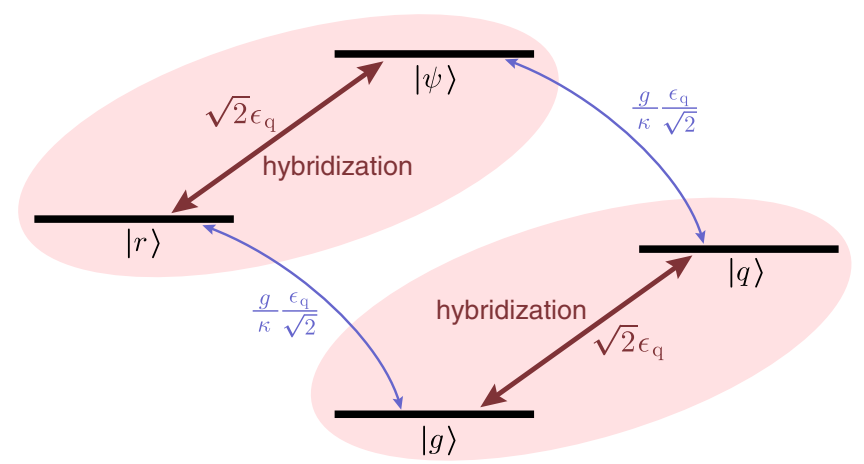

FIG. 10. Effective four-level model explaining the anomalous resonance. The transitions $|g\rangle \leftrightarrow|q\rangle$ and $|r\rangle \leftrightarrow|\psi\rangle$ strongly hybridize two pairs of states. The anomalous resonance results from transitions between the two hybridized doublets. Since the energy separation between hybridized doublets depends on drive strength $\epsilon$, so does the position of the anomalous resonance. 
Because of the energy differences between states within each pair, hybridization is only partial. The two emerging hybridized states relevant to the anomalous resonance have significant overlap with the ground state $|g\rangle$ in one case and the two-excitation state $|\psi\rangle$ in the other case. The resonance C can be approximately viewed as a resonance between these two hybridized states. Note that the degree of hybridization critically depends on the effective drive strength $\epsilon_{\mathrm{q}}$, which is in turn proportional to the drive strength $\epsilon$. As a consequence, the energy separation between the two relevant hybridized states depends on drive strength as observed in Fig. 9(b).

The generalization of the effective four-level model to periodic chains with a larger number of sites $N$ is difficult because of the proliferation of degeneracies among eigenstates of $\mathrm{H}^{\prime}$ in the absence of a drive. Based on our perturbative calculations, we find a clear trend of diminishing resonance strength with increasing number of sites [Fig. 9(a)]. The anomalous resonance $\mathrm{C}$ hence provides an interesting example of an interaction-induced feature limited to a small $N$, which should be accessible in experiments with Jaynes-Cummings chains of only a few sites.

\section{CONCLUSION}

We have extended the general Lindblad perturbation framework to include resummation of an infinite subset of perturbative corrections. We have formulated the scheme at a general level, emphasizing that this is not limited to a particular open quantum system but benefits a variety of problems in Markovian quantum systems amenable to perturbative treatment. For the examples we have investigated, we find that the series resummation can significantly improve the accuracy of the perturbative treatment.

We have applied perturbation theory with resummation to a specific model of an open quantum lattice, the open Jaynes-Cummings lattice, and have introduced a diagrammatic representation systematically organizing the contributing terms. For small lattices, we find very good agreement with exact results, which we obtained by extensive quantum trajectory simulations for an interesting parameter regime near resonance. Our perturbative treatment is capable of predicting steady-state observables for both finite and infinite Jaynes-Cummings lattices with different lattice geometries and dimensionalities - thus including settings that may not be easily accessible by other methods.

The capability of obtaining reliable results beyond exactly solvable limits of open quantum lattices is particularly promising as a method for validating experimental implementations of quantum simulators. Concrete realizations of open quantum lattices are currently being investigated in the circuit QED architecture [64].

Finally, questions that warrant future investigations regard the relation between mean-field approximations, suitable choices of resummation, and additional justification of the resummation series for specific physics systems within the presented formalism. We note that resummation schemes are also common within the framework of Keldysh Green's functions. However, handling (pseudo) spin degrees of freedom tends to be more challenging within this method. It will be interesting to compare and relate results obtainable with both our method and the Keldysh technique in the context of simpler systems such as the open Bose-Hubbard lattice. Further investigations into spectra of Liouvillians, handling cases of degeneracies of the Liouvillian spectrum, and extending resummation to time-dependent perturbation theory offer exciting perspectives for the study of dissipative phase transitions in open quantum systems.

\section{ACKNOWLEDGMENTS}

We acknowledge valuable discussions with Guanyu Zhu and Joshua Dempster, and thank Ataç İmamoğlu, Juan José García-Ripoll, and Mark I. Dykman for pointing out useful references developing and applying Lindblad perturbation theory. This research was supported in part through the computational resources and staff contributions provided for the Quest High Performance Computing Facility at Northwestern University, by the NSF under Grant No. PHY-1055993 (A. C. Y. L. and J. K.) and under Grant No. PHY11-25915 (J. K.), and by the South African Research Chair Initiative of the Department of Science and Technology and National Research Foundation (F. P.).

\section{APPENDIX A: DETAILS OF RESUMMATION}

This appendix provides the proof that powers of the $\mathbb{U}$ superoperator can be written as [Eq. (28)],

$$
\mathbb{U}^{j}=\sum_{k=0}^{j} \mathbb{s}_{j-k} \mathbb{\mathbb { T }}_{k},
$$

where the prefactor $\mathbb{S}_{j-k}$ consists of all possible combinations of $\mathbb{S}$ superoperators of total order $j-k$. The definitions of the involved $\mathbb{T}$ superoperator and $\mathbb{S}$ superoperator [Eqs. (18) and (21)] are

$$
\begin{gathered}
\mathbb{S}_{1}=0, \quad \mathbb{T}_{1}=\mathbb{U}, \\
\mathbb{T}_{j-1} \mathbb{U}=\mathbb{S}_{j}+\mathbb{T}_{j}, \quad\left(\mathbb{S}_{j}\right)_{\mu \nu}=\delta_{\mu \nu}\left(\mathbb{T}_{j-1} \mathbb{U}\right)_{\mu \nu} .
\end{gathered}
$$

We further define $s_{0}=\mathbb{T}_{0}=\mathbb{1}$.

We prove Eq. (A1) by mathematical induction. The $\mathbb{U}^{1}$ case clearly satisfies Eq. (A1),

$$
\mathbb{U}^{1}=\mathbb{T}_{1}=\mathbb{S}_{1} \mathbb{1}+\mathbb{\mathbb { T }} \mathbb{\mathbb { T }}_{1}=\mathbb{S}_{1} \mathbb{\mathbb { T }}_{0}+\mathbb{s}_{0} \mathbb{\mathbb { T }}_{1} .
$$

Assume that Eq. (A1) holds up to power $j-1$, i.e.,

$$
\mathbb{U}^{j-1}=\sum_{k=0}^{j-1} \mathbb{S}_{k} \mathbb{\mathbb { T }}_{j-1-k} .
$$


Then, the decomposition rule (23) yields

$$
\mathbb{U}^{j}=\mathbb{U}^{j-1} \mathbb{U}=\sum_{k=0}^{j-1} \mathbb{s}_{k} \mathbb{T}_{j-1-k} \mathbb{U} .
$$

The product $\mathbb{\mathbb { T }}_{j-1-k} \mathbb{U}$ is separated according to Eq. (A3), i.e., $\mathbb{T}_{j-1-k} \mathbb{U}=\mathbb{S}_{j-k}+\mathbb{T}_{j-k}$, so we obtain

$$
\mathbb{U}^{j}=\sum_{k=0}^{j-1} \mathbb{S}_{k} \mathbb{S}_{j-k}+\sum_{k=0}^{j-1} \mathbb{S}_{k} \mathbb{\mathbb { T }}_{j-k} .
$$

The first sum, $\sum_{k=0}^{j-1} \mathbb{S}_{k} \mathbb{S}_{j-k}$, consists of all products of $\mathbb{S}$ superoperators with combined order $j$, i.e., $\sum_{k=0}^{j-1} \mathbb{S}_{k} \mathbb{S}_{j-k}=\mathbb{s}_{j}$. As a result, it follows that

$$
\mathbb{U}^{j}=\mathbb{s}_{j} \mathbb{\mathbb { T }}_{0}+\sum_{k=0}^{j-1} \mathbb{s}_{k} \mathbb{\mathbb { T }}_{j-k}=\sum_{k=0}^{j} \mathbb{s}_{k} \mathbb{\mathbb { T }}_{j-k},
$$

which concludes the proof.

\section{APPENDIX B: COMPUTATIONAL COST FOR PERTURBATIVE CALCULATIONS}

The computational cost in calculating perturbative results for the open Jaynes-Cummings lattice primarily stems from summation over matrix elements of the $\mathbb{U}^{\mathbf{k r}}$ superoperator. For each summation, the number of terms is given by $N$, which corresponds to either the number of qubits or photon modes. The number of necessary summations for rank- $j$ corrections is given by $2 j$ [see Eqs. (45) and (46)]. As a result, the overall cost scales algebraically with the number of sites, namely, approximately $N^{2 j}$. Results for infinite lattices hinge upon the possibility to carry out summations analytically for specific cases, such as for leading-rank corrections of infinite JaynesCummings lattices with periodic-chain or global-coupling geometry.

[1] M. C. Gutzwiller, Effect of Correlation on the Ferromagnetism of Transition Metals, Phys. Rev. Lett. 10, 159 (1963).

[2] J. Hubbard, Electron Correlations in Narrow Energy Bands, Proc. R. Soc. A 276, 238 (1963).

[3] J. Hubbard, Electron Correlations in Narrow Energy Bands. II. The Degenerate Band Case, Proc. R. Soc. A 277, 237 (1964).

[4] M. P. A. Fisher, P. B. Weichman, G. Grinstein, and D. S. Fisher, Boson Localization and the Superfluid-Insulator Transition, Phys. Rev. B 40, 546 (1989).

[5] W. Krauth, M. Caffarel, and J.-P. Bouchaud, Gutzwiller Wave Function for a Model of Strongly Interacting Bosons, Phys. Rev. B 45, 3137 (1992).

[6] R. Orbach, Linear Antiferromagnetic Chain with Anisotropic Coupling, Phys. Rev. 112, 309 (1958).

[7] P. W. Anderson, Resonating Valence Bonds: A New Kind of Insulator?, Mater. Res. Bull. 8, 153 (1973).
[8] S. Takei and Y. B. Kim, Nonequilibrium-Induced MetalSuperconductor Quantum Phase Transition in Graphene, Phys. Rev. B 78, 165401 (2008).

[9] N. Sedlmayr, J. Ren, F. Gebhard, and J. Sirker, Closed and Open System Dynamics in a Fermionic Chain with a Microscopically Specified Bath: Relaxation and Thermalization, Phys. Rev. Lett. 110, 100406 (2013).

[10] W.-R. Lee and K. Park, Dielectric Breakdown via Emergent Nonequilibrium Steady States of the Electric-Field-Driven Mott Insulator, Phys. Rev. B 89, 205126 (2014).

[11] I. Carusotto, D. Gerace, H. E. Türeci, S. De Liberato, C. Ciuti, and A. Imamoğlu, Fermionized Photons in an Array of Driven Dissipative Nonlinear Cavities, Phys. Rev. Lett. 103, 033601 (2009).

[12] T. Ozawa and I. Carusotto, Anomalous and Quantum Hall Effects in Lossy Photonic Lattices, Phys. Rev. Lett. 112, 133902 (2014).

[13] M. Hafezi, Measuring Topological Invariants in Photonic Systems, Phys. Rev. Lett. 112, 210405 (2014).

[14] J. Raftery, D. Sadri, S. Schmidt, H. E. Türeci, and A. A. Houck, Observation of a Dissipation-Induced Classical to Quantum Transition, Phys. Rev. X 4, 031043 (2014).

[15] E. Kapit, M. Hafezi, and S. H. Simon, Induced SelfStabilization in Fractional Quantum Hall States of Light, Phys. Rev. X 4, 031039 (2014).

[16] V. Peano, C. Brendel, M. Schmidt, and F. Marquardt, Topological Phases of Sound and Light, Phys. Rev. X 5, 031011 (2015).

[17] T. Gao, E. Estrecho, K. Y. Bliokh, T. C. H. Liew, M. D. Fraser, S. Brodbeck, M. Kamp, C. Schneider, S. Höfling, Y. Yamamoto, F. Nori, Y. S. Kivshar, A. G. Truscott, R. G. Dall, and E. A. Ostrovskaya, Observation of Non-Hermitian Degeneracies in a Chaotic Exciton-Polariton Billiard, Nature (London) 526, 554 (2015).

[18] M. H. Devoret and R. J. Schoelkopf, Superconducting Circuits for Quantum Information: An Outlook, Science 339, 1169 (2013).

[19] S. Boixo, T. F. Rønnow, S. V. Isakov, Z. Wang, D. Wecker, D. A. Lidar, J. M. Martinis, and M. Troyer, Evidence for Quantum Annealing with More Than One Hundred Qubits, Nat. Phys. 10, 218 (2014).

[20] M. Schuld, I. Sinayskiy, and F. Petruccione, The Quest for a Quantum Neural Network, Quantum Inf. Process. 13, 2567 (2014).

[21] D. S. Pavlichin and H. Mabuchi, Photonic Circuits for Iterative Decoding of a Class of Low-Density Parity-Check Codes, New J. Phys. 16, 105017 (2014).

[22] N. H. Nickerson, J. F. Fitzsimons, and S. C. Benjamin, Freely Scalable Quantum Technologies Using Cells of 5-to-50 Qubits with Very Lossy and Noisy Photonic Links, Phys. Rev. X 4, 041041 (2014).

[23] C. Aron, M. Kulkarni, and H. E. Türeci, Steady-State Entanglement of Spatially Separated Qubits via Quantum Bath Engineering, Phys. Rev. A 90, 062305 (2014).

[24] K. Baumann, C. Guerlin, F. Brennecke, and T. Esslinger, Dicke Quantum Phase Transition with a Superfluid Gas in an Optical Cavity, Nature (London) 464, 1301 (2010).

[25] D. Nagy, G. Szirmai, and P. Domokos, Critical Exponent of a Quantum-Noise-Driven Phase Transition: The OpenSystem Dicke Model, Phys. Rev. A 84, 043637 (2011). 
[26] T. Grujic, S. R. Clark, D. Jaksch, and D. G. Angelakis, Non-Equilibrium Many-Body Effects in Driven Nonlinear Resonator Arrays, New J. Phys. 14, 103025 (2012).

[27] T. E. Lee, H. Häffner, and M. C. Cross, Collective Quantum Jumps of Rydberg Atoms, Phys. Rev. Lett. 108, 023602 (2012).

[28] E. M. Kessler, G. Giedke, A. Imamoglu, S. F. Yelin, M. D. Lukin, and J. I. Cirac, Dissipative Phase Transition in a Central Spin System, Phys. Rev. A 86, 012116 (2012).

[29] F. Nissen, S. Schmidt, M. Biondi, G. Blatter, H. E. Türeci, and J. Keeling, Nonequilibrium Dynamics of Coupled Qubit-Cavity Arrays, Phys. Rev. Lett. 108, 233603 (2012).

[30] G. Kulaitis, F. Krüger, F. Nissen, and J. Keeling, Disordered Driven Coupled Cavity Arrays: Nonequilibrium Stochastic Mean-Field Theory, Phys. Rev. A 87, 013840 (2013).

[31] L. M. Sieberer, S. D. Huber, E. Altman, and S. Diehl, Dynamical Critical Phenomena in Driven-Dissipative Systems, Phys. Rev. Lett. 110, 195301 (2013).

[32] L. M. Sieberer, S. D. Huber, E. Altman, and S. Diehl, Nonequilibrium Functional Renormalization for DrivenDissipative Bose-Einstein Condensation, Phys. Rev. B 89, 134310 (2014).

[33] J. Jin, D. Rossini, M. Leib, M. J. Hartmann, and R. Fazio, Steady-State Phase Diagram of a Driven QED-Cavity Array with Cross-Kerr Nonlinearities, Phys. Rev. A 90, 023827 (2014).

[34] A. Le Boité, G. Orso, and C. Ciuti, Bose-Hubbard Model: Relation between Driven-Dissipative Steady States and Equilibrium Quantum Phases, Phys. Rev. A 90, 063821 (2014).

[35] J. Klinder, H. Keßler, M. Wolke, L. Mathey, and A. Hemmerich, Dynamical Phase Transition in the Open Dicke Model, Proc. Natl. Acad. Sci. U.S.A. 112, 3290 (2015).

[36] U. Naether, F. Quijandría, J. J. García-Ripoll, and D. Zueco, Stationary Discrete Solitons in a Driven Dissipative BoseHubbard Chain, Phys. Rev. A 91, 033823 (2015).

[37] J. Marino and S. Diehl, Driven Markovian Quantum Criticality, Phys. Rev. Lett. 116, 070407 (2016).

[38] J. M. Zeuner, M. C. Rechtsman, Y. Plotnik, Y. Lumer, S. Nolte, M. S. Rudner, M. Segev, and A. Szameit, Observation of a Topological Transition in the Bulk of a NonHermitian System, Phys. Rev. Lett. 115, 040402 (2015).

[39] V. Fernández-Hurtado, J. Mur-Petit, J. José García-Ripoll, and R. A. Molina, Lattice Scars: Surviving in an Open Discrete Billiard, New J. Phys. 16, 035005 (2014).

[40] H.-P. Breuer and F. Petruccione, The Theory of Open Quantum Systems (Oxford University Press, New York, 2007).

[41] R. Alicki and K. Lendi, Quantum Dynamical Semigroups and Applications (Springer, New York, 2007), p. 142.

[42] H. J. Briegel and B. G. Englert, Quantum Optical Master Equations: The Use of Damping Bases, Phys. Rev. A 47, 3311 (1993).

[43] J. M. Torres, Closed-Form Solution of Lindblad Master Equations without Gain, Phys. Rev. A 89, 052133 (2014).

[44] M. B. Plenio and P. L. Knight, The Quantum-Jump Approach to Dissipative Dynamics in Quantum Optics, Rev. Mod. Phys. 70, 101 (1998).
[45] F. Verstraete, J. J. García-Ripoll, and J. I. Cirac, Matrix Product Density Operators: Simulation of Finite-Temperature and Dissipative Systems, Phys. Rev. Lett. 93, 207204 (2004).

[46] M. Zwolak and G. Vidal, Mixed-State Dynamics in OneDimensional Quantum Lattice Systems: A Time-Dependent Superoperator Renormalization Algorithm, Phys. Rev. Lett. 93, 207205 (2004).

[47] P. Degenfeld-Schonburg and M. J. Hartmann, SelfConsistent Projection Operator Theory for Quantum Many-Body Systems, Phys. Rev. B 89, 245108 (2014).

[48] H. Weimer, Variational Principle for Steady States of Dissipative Quantum Many-Body Systems, Phys. Rev. Lett. 114, 040402 (2015).

[49] J. Cui, J. I. Cirac, and M. C. Bañuls, Variational Matrix Product Operators for the Steady State of Dissipative Quantum Systems, Phys. Rev. Lett. 114, 220601 (2015).

[50] M. Hafezi, P. Adhikari, and J. M. Taylor, A Chemical Potential for Light, Phys. Rev. B 92, 174305 (2015).

[51] D. L. Underwood, W. E. Shanks, J. Koch, and A. A. Houck, Low-Disorder Microwave Cavity Lattices for Quantum Simulation with Photons, Phys. Rev. A 86, 023837 (2012).

[52] A. A. Houck, H. E. Türeci, and J. Koch, On-Chip Quantum Simulation with Superconducting Circuits, Nat. Phys. 8, 292 (2012).

[53] S. Schmidt and J. Koch, Circuit QED Lattices: Towards Quantum Simulation with Superconducting Circuits, Ann. Phys. (Amsterdam) 525, 395 (2013).

[54] J. I. Cirac and P. Zoller, Goals and Opportunities in Quantum Simulation, Nat. Phys. 8, 264 (2012).

[55] J. J. García-Ripoll, S. Dürr, N. Syassen, D. M. Bauer, M. Lettner, G. Rempe, and J. I. Cirac, Dissipation-Induced Hard-Core Boson Gas in an Optical Lattice, New J. Phys. 11, 013053 (2009).

[56] F. Benatti, A. Nagy, and H. Narnhofer, Asymptotic Entanglement and Lindblad Dynamics: A Perturbative Approach, J. Phys. A 44, 155303 (2011).

[57] Z. Maizelis, M. Rudner, and M. I. Dykman, Vibration Multistability and Quantum Switching for Dispersive Coupling, Phys. Rev. B 89, 155439 (2014).

[58] A. C. Y. Li, F. Petruccione, and J. Koch, Perturbative Approach to Markovian Open Quantum Systems, Sci. Rep. 4, 4887 (2014).

[59] K. L. Hur, Entanglement Entropy, Decoherence, and Quantum Phase Transition of a Dissipative Two-Level System, Ann. Phys. (Amsterdam) 323, 2208 (2007).

[60] M. J. Hartmann, Polariton Crystallization in Driven Arrays of Lossy Nonlinear Resonators, Phys. Rev. Lett. 104, 113601 (2010).

[61] C. Joshi, F. Nissen, and J. Keeling, Quantum Correlations in the One-Dimensional Driven Dissipative XY Model, Phys. Rev. A 88, 063835 (2013).

[62] J. Ruiz-Rivas, E. del Valle, C. Gies, P. Gartner, and M. J. Hartmann, Spontaneous Collective Coherence in Driven Dissipative Cavity Arrays, Phys. Rev. A 90, 033808 (2014).

[63] H. J. Carmichael, Breakdown of Photon Blockade: A Dissipative Quantum Phase Transition in Zero Dimensions, Phys. Rev. X 5, 031028 (2015).

[64] A. A. Houck (unpublished). 
[65] T. Prosen, Third Quantization: A General Method to Solve Master Equations for Quadratic Open Fermi Systems, New J. Phys. 10, 043026 (2008).

[66] T. Prosen and I. Pižorn, Quantum Phase Transition in a Far-from-Equilibrium Steady State of an XY Spin Chain, Phys. Rev. Lett. 101, 105701 (2008).

[67] T. Prosen, Spectral Theorem for the Lindblad Equation for Quadratic Open Fermionic Systems, J. Stat. Mech. (2010) P07020.

[68] T. Prosen and T. H. Seligman, Quantization Over Boson Operator Spaces, J. Phys. A 43, 392004 (2010).

[69] A. A. Dzhioev and D. S. Kosov, Super-Fermion Representation of Quantum Kinetic Equations for the Electron Transport Problem, J. Chem. Phys. 134, 044121 (2011).

[70] T. Prosen, Exact Nonequilibrium Steady State of a Strongly Driven Open XXZ Chain, Phys. Rev. Lett. 107, 137201 (2011).

[71] I. Pižorn, One-Dimensional Bose-Hubbard Model Far from Equilibrium, Phys. Rev. A 88, 043635 (2013).

[72] M. V. Medvedyeva, M. T. Čubrović, and S. Kehrein, Dissipation-Induced First-Order Decoherence Phase Transition in a Noninteracting Fermionic System, Phys. Rev. B 91, 205416 (2015).

[73] J. Ningyuan, C. Owens, A. Sommer, D. Schuster, and J. Simon, Time- and Site-Resolved Dynamics in a Topological Circuit, Phys. Rev. X 5, 021031 (2015).

[74] M. Knap, E. Arrigoni, W. von der Linden, and J. H. Cole, Emission Characteristics of Laser-Driven Dissipative Coupled-Cavity Systems, Phys. Rev. A 83, 023821 (2011).

[75] A. Nunnenkamp, J. Koch, and S. M. Girvin, Synthetic Gauge Fields and Homodyne Transmission in JaynesCummings Lattices, New J. Phys. 13, 095008 (2011).

[76] M. J. Bhaseen, J. Mayoh, B. D. Simons, and J. Keeling, Dynamics of Nonequilibrium Dicke Models, Phys. Rev. A 85, 013817 (2012).
[77] G. Kónya, D. Nagy, G. Szirmai, and P. Domokos, FiniteSize Scaling in the Quantum Phase Transition of the OpenSystem Dicke Model, Phys. Rev. A 86, 013641 (2012).

[78] L. J. Zou, D. Marcos, S. Diehl, S. Putz, J. Schmiedmayer, J. Majer, and P. Rabl, Implementation of the Dicke Lattice Model in Hybrid Quantum System Arrays, Phys. Rev. Lett. 113, 023603 (2014).

[79] J. Jin, D. Rossini, R. Fazio, M. Leib, and M. J. Hartmann, Photon Solid Phases in Driven Arrays of Nonlinearly Coupled Cavities, Phys. Rev. Lett. 110, 163605 (2013).

[80] M. Schiró, C. Joshi, M. Bordyuh, R. Fazio, J. Keeling, and H.E. Türeci, Exotic Attractors of the Nonequilibrium Rabi-Hubbard Model, Phys. Rev. Lett. 116, 143603 (2016).

[81] J. D. Cresser, Thermal Equilibrium in the Jaynes-Cummings Model, J. Mod. Opt. 39, 2187 (1992).

[82] The Liouville superoperator $\mathbb{E}$ is not Hermitian, so completeness of the set of eigenstates is not guaranteed, though heuristically it is very common for $\mathbb{L}$.

[83] If the unperturbed eigenstates are not complete, a generalized inverse $[56,58]$ or projection techniques $[28,55]$ must be used.

[84] For an infinite system, $N \gg 1$ is the auxiliary lattice size involved in applying Born-von Karmann periodic boundary conditions.

[85] B.-G. Englert and G. Morigi, in Coherent Evolution in Noisy Environments, Lecture Notes in Physics, Vol. 611, edited by A. Buchleitner and K. Hornberger (Springer, Berlin, Heidelberg, 2002), pp. 55-106.

[86] To quickly confirm the tracelessness, refer to the orthonormality condition (9) and note that the left eigenstate corresponding to the steady state is the identity $\left(\breve{\rho}^{[0]} \mid=1\right.$.

[87] G. Zhu, S. Schmidt, and J. Koch, Dispersive Regime of the Jaynes-Cummings and Rabi Lattice, New J. Phys. 15, 115002 (2013). 